\title{
Symbiosis, virulence and natural-product biosynthesis in entomopathogenic bacteria are regulated by a small RNA
}

\author{
Nick Neubacher $\circledR^{1,10}$, Nicholas J. Tobias $\rrbracket^{1,2,3,10}$, Michaela Huber ${ }^{4,5,6,10}$, Xiaofeng Cai ${ }^{1}$, \\ Timo Glatter7, Sacha J. Pidot $\mathbb{1}^{8}{ }^{8}$, Timothy P. Stinear ${ }^{8}{ }^{8}$, Anna Lena Lütticke ${ }^{1}$, Kai Papenfort $\mathbb{B}^{4,5}$ and \\ Helge B. Bode $\mathbb{D}^{1,2,3,9 凶}$
}

\begin{abstract}
Photorhabdus and Xenorhabdus species have mutualistic associations with nematodes and an entomopathogenic stage $^{1,2}$ in their life cycles. In both stages, numerous specialized metabolites are produced that have roles in symbiosis and virulence $e^{3,4}$. Although regulators have been implicated in the regulation of these specialized metabolites ${ }^{3,4}$, how small regulatory RNAs (sRNAs) are involved in this process is not clear. Here, we show that the Hfq-dependent sRNA, ArcZ, is required for specialized metabolite production in Photorhabdus and Xenorhabdus. We discovered that ArcZ directly base-pairs with the mRNA encoding HexA, which represses the expression of specialized metabolite gene clusters. In addition to specialized metabolite genes, we show that the ArcZ regulon affects approximately $15 \%$ of all transcripts in Photorhabdus and Xenorhabdus. Thus, the ArcZ sRNA is crucial for specialized metabolite production in Photorhabdus and Xenorhabdus species and could become a useful tool for metabolic engineering and identification of commercially relevant natural products.
\end{abstract}

Regulation via trans-encoded small RNAs (sRNAs) typically occurs by imperfect base-pairing of sRNAs with their messenger-RNA targets and can be mediated by RNA chaperones such as Hfq and ProQ ${ }^{5,6}$. RNA duplexes are usually short (6-12 nucleotides) and can result in conformational changes in the RNA secondary structure with various regulatory outcomes ${ }^{7}$. The RNA chaperone Hfq is highly conserved throughout the bacterial kingdom ${ }^{8}$. Several complex phenotypes have been attributed to Hfq, which performs its regulatory roles by stabilizing sRNAs and/or mRNAs, mediating base-pairing of sRNAs and their targets, modulation of mRNA translation ${ }^{8}$ as well as accelerating the degradation of sRNAs and their targets ${ }^{5}$. The expression of sRNAs is highly dynamic, with sRNA profiles in Salmonella shown to be strongly dependent on the bacterial growth phase ${ }^{9}$. ArcZ is one of the few Hfq-bound sRNAs whose expression remains relatively constant in Salmonella throughout the growth phases, constituting approximately $7-12 \%$ of all reads identified by Hfq co-immunoprecipitation experiments ${ }^{9}$. ArcZ is transcribed as a 129-nucleotide primary transcript (Fig. 1a) and processed into a stable short form (approximately 50 nucleotides) ${ }^{9-11}$. The processed short form of ArcZ activates rpoS translation directly and inhibits the expression of several other genes ${ }^{11,12}$. In Escherichia coli, the expression of $\operatorname{arc} Z$ is repressed by the ArcA-ArcB two-component system under anaerobic conditions. In a negative feedback loop, $\operatorname{arc} Z$ represses and is repressed by $\operatorname{arc} B$ transcription ${ }^{11}$. Although there is a wealth of research on ArcZ in E. coli and Salmonella ${ }^{9-11}$, its function in other bacteria remains unclear.

Specialized metabolites (SMs) in bacteria are often responsible for ecologically important activities ${ }^{13}$. In the case of Xenorhabdus and Photorhabdus, SMs play an essential role in cross-kingdom interactions with nematodes, various insects as well as bacterial and fungal species competing for the same food source ${ }^{14}$. Our earlier work on Photorhabdus showed that the deletion of $h f q$ results in severe perturbation of gene networks, including several key regulators ${ }^{4}$. This leads to an overall decrease in SM production and a failure of the bacteria to support their obligate symbiosis with nematodes. Despite SMs playing a central role in the life cycle of the symbiosis, the exact ecological functions of many of these compounds has remained unknown. Notable advances towards the identification of the bioactivities of many of the SMs-with assigned functions including cell-cell communication (photopyrones and dialkylresorcinols $^{15,16}$ ), nematode development (isopropylstilbene ${ }^{17}$ ), defence against food competitors (isopropylstilbene and rhabdopeptides ${ }^{17,18}$ ) or insect pathogenicity (rhabduscin, rhabdopeptides and glidobactin ${ }^{18-20}$ ) - have been made in recent years. However, understanding the full potential of SMs in these bacteria is still hampered by a somewhat limited understanding of when individual SMs are produced and their regulation in general. The regulators Hfa, HexA (also LrhA), LeuO and Lrp have so far been implicated in the regulation of SMs in Photorhabdus and Xenorhabdus s, $^{3,21,22}$. Deletion of $h f q$ in Photorhabdus results in complex regulatory changes, including a strong upregulation of HexA, a known repressor of SM production $^{4}$. Consequently, SM production is completely abolished in this strain and nematode development was severely restricted.

Given the overlapping life cycles and niche occupation, we hypothesized that the deletion of $h f q$ in Xenorhabdus would have

\footnotetext{
'Molekulare Biotechnologie, Fachbereich Biowissenschaften, Goethe-Universität Frankfurt, Frankfurt, Germany. ${ }^{2}$ LOEWE Center for Translational Biodiversity in Genomics (TBG), Frankfurt, Germany. ${ }^{3}$ Senckenberg Gesellschaft für Naturforschung, Frankfurt, Germany. ${ }^{4}$ Institute of Microbiology, Friedrich Schiller University Jena, Jena, Germany. ${ }^{5}$ Microverse Cluster, Friedrich Schiller University Jena, Jena, Germany. ${ }^{6}$ Faculty of Biology I, Ludwig-Maximilians-University of Munich, Martinsried, Germany. ${ }^{7}$ Core Facility for Mass Spectrometry and Proteomics, Max Planck Institute for Terrestrial Microbiology, Marburg, Germany. ${ }^{8}$ Department of Microbiology and Immunology, Peter Doherty Institute for Infection and Immunity, University of Melbourne, Melbourne, Victoria, Australia. ${ }^{9}$ Buchmann Institute for Molecular Life Sciences, Goethe-Universität Frankfurt, Frankfurt, Germany. ${ }^{10}$ These authors contributed equally: Nick Neubacher, Nicholas J. Tobias, Michaela Huber. ${ }^{凶}$-mail: h.bode@bio.uni-frankfurt.de
} 


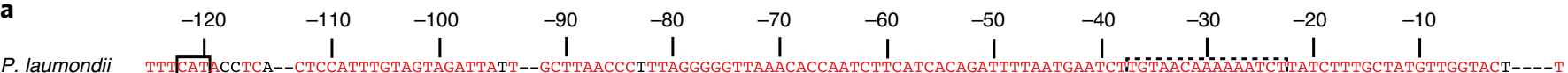
P. temperata TTICAThGCTCA--CTCCATTTGTAGTAGATTATT--GCTTAACCCTTTAGGGGGTTAAACACCAATCTTCATCACAGATTTTAATGAATCTITGTAACAAAAAATCT'TATCTTTGCTATGTTGGTACT----T $P$ asymbiotica TII CATCCTCT-DTCCATTTGTATA $X$ nematophila $X$. hOVioni

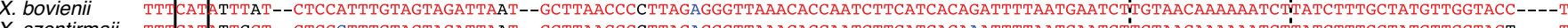
$X$. szentirmaii TTI CATATTCCT--CTCCGTTTGTAGTAGATTAAT--GCTTAACCCCTTAGAGGGTTAAACACCAATCTTCATCACAAATTTTAATGAATCTY'TGTAACAAAAAATCTIATCTTTGCTATGTTGGTACT----T S. marcescens TTICAT CACTCTTACTCCTGTCGCTGCTGTCAACT--GGTTCGTGCTCTGAGTTGACAAACACTAATCTACATCACACATTTTAATGAATCTICTAACAAGAGCGCITACATTTGCTATGTTGTTGGC----T E. amylovora TCGTTCAAATTG-ATTCCTTGCTTGACTAAATGCTCAAATTCGGGTCTCCTGTTGAAAGACGCCGTTCAACATCACACATTTTAATGAATCTGGTAACATGTGATGAÁAGATTTGCTAAGCTAGTAACACGAT E. coli CTT CATTGTGAT-----CATCCTTCGCAATTGACTGAAACACATTAATTTTTTTAATAAA-AATGGTACGCATCACACATTTAACTGATTCATGTAACAAATCATTTAAGTTTTGCTATCTTAACTGC--

S. typhimurium -TILATGGTGAA-----CATCTCCTGCAACCGACTCAAGCCCATTAATAATTTTGATTAA-AATGCTACGCTTCACACATTTAAATGATTCATGTAACAAATCATTTAGGATTTGCTATCTTAACTGC----$\longleftarrow$ elbB $\quad$ ArcA binding site

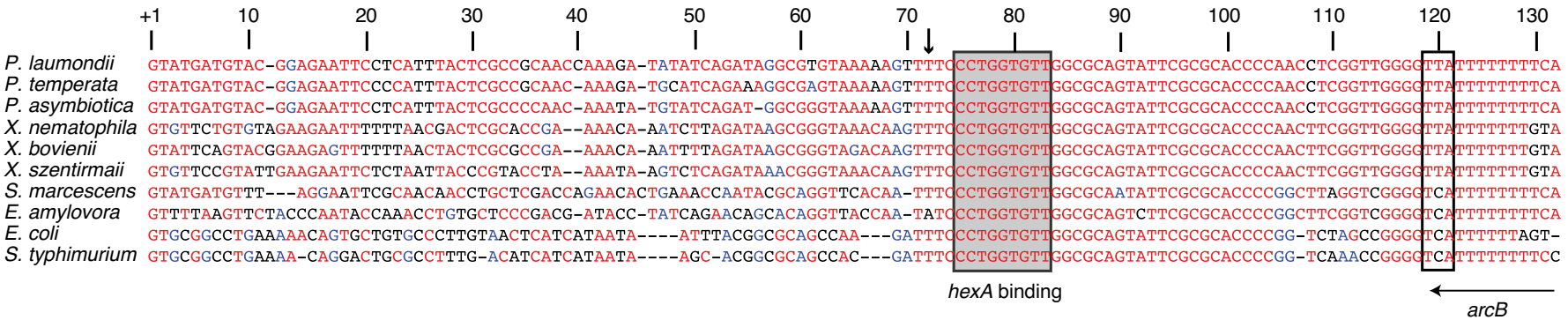

b

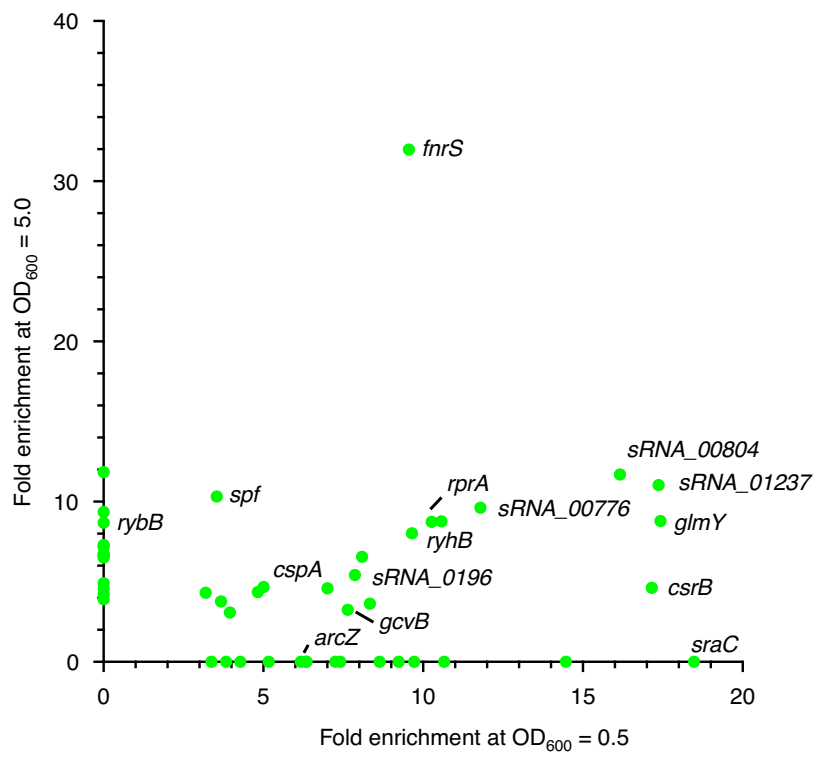

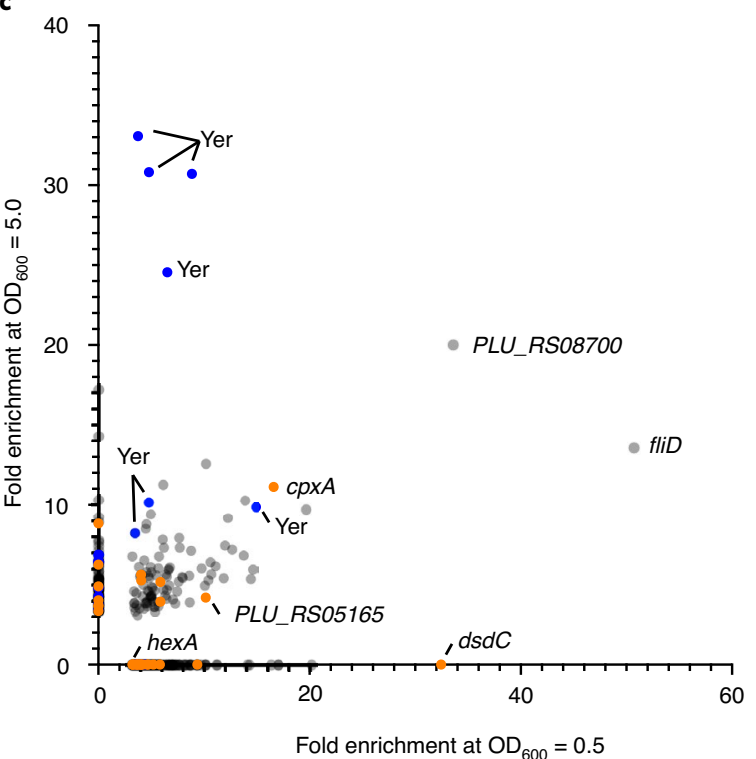

Fig. 1 | ArcZ is a conserved Hfq-binding sRNA. a, Alignment of arcZ sequences from P. laumondii TTO1, Photorhabdus temperata, Photorhabdus asymbiotica, Xenorhabdus nematophila, Xenorhabdus bovienii, X. szentirmaii, Serratia marcescens, Erwinia amylovora, E. coli K12 and Salmonella typhimurium LT2. The numbers refer to the nucleotide positions of the $P$. laumondii sequence. The +1 indicates the transcriptional start of the 129-nucleotide $\operatorname{arc} Z$ sequence. The start codon for elbB and stop codon for $\operatorname{arc} B,-10$ and -35 binding regions as well as the conserved ArcA binding region ${ }^{11}$ and the region of base-pairing to hex $A$ are indicated. The site of ArcZ cleavage is indicated by an arrow. $\mathbf{b}$, Enrichment, determined by RIP-seq, in regions of sRNAs. c, Comparison of the mRNAs in a strain containing $\mathrm{Hfq}^{3 \times F \mathrm{LAG}}$ and the untagged control strain at both optical densities. Yer, yersiniabactin-related genes. For a complete list of the enriched regions see Supplementary Tables 4 and 5. The blue dots represent SM-related mRNAs and orange dots represent mRNAs associated with annotated regulators.

a similar effect on the production of SMs and the transcriptome. We confirmed this in Xenorhabdus szentirmaii DSM16338 using both high-performance liquid chromatography (HPLC) coupled with tandem mass spectrometry (MS/MS) and RNA sequencing (Supplementary Note 1 and Supplementary Table 1). We investigated the Hfq binding partners to further elucidate the mechanism of SM regulation. To this end, we sequenced both X. szentirmaii and Photorhabdus laumondii using a sRNA sequencing protocol and combined this with Cappable-seq data to globally annotate the transcriptional start sites belonging to coding sequences or potential previously undescribed sRNAs (Supplementary Note 2 and Supplementary Table 2). We confirmed the expression of several of these sRNAs by northern blot analysis (Extended Data Figs. 1 and 2). To identify RNA-protein interactions on a global scale, we next employed RNA immunoprecipitation, followed by high-throughput sequencing (RIP-seq) using chromosomally produced $\mathrm{Hfq}^{3 \times \mathrm{FLAG}}$ protein as bait. We performed these experiments at two different cell densities (that is, at an optical density (OD) ${ }_{600}$ of 0.5 and 5.0; see Supplementary Table 3 for a full list of the ODs from different experiments). From the corresponding sequencing data, we first identified regions of 5 base pairs (bp) or more that were enriched in our tagged Hfq strain (Methods). We then searched for sRNAs that were specifically enriched in the tagged samples compared with the untagged samples. We identified a total of 37 binding 
sites in annotated sRNAs ( 35 unique sRNAs) at an $\mathrm{OD}_{600}$ of 0.5 and 37 binding sites ( 34 unique) at an $\mathrm{OD}_{600}$ of 5.0 that were enriched by at least threefold in both replicates (Fig. 1b and Supplementary Table 4). During early exponential growth, 11 sRNAs (of 35) that have been described to associate with $\mathrm{Hfq}$ in other species were identified, whereas 10 (of 34) are known from those that were enriched at an $\mathrm{OD}_{600}$ of 5.0. As a second step, we examined the mRNAs enriched in the data. At an $\mathrm{OD}_{600}$ of 5.0, 402 mRNAs and 32 annotated $5^{\prime}$ untranslated regions (UTRs) were identified to associate with Hfq. A total of 1,003 mRNAs and $295^{\prime}$ UTRs were detected at an $\mathrm{OD}_{600}$ of 0.5 , (Fig. 1c and Supplementary Table 5).

We hypothesized that the Hfq RIP-seq analysis performed would allow us to identify key sRNAs involved in SM repression. However, our analysis identified $>50$ potential Hfq-binding sRNAs (across both ODs; Fig. 1b). We therefore constructed a transposon-mutant library using pSAM-BT_Kan instead of deleting each sRNA individually (Methods and Supplementary Note 3) and searched for phenotypes consistent with that of the strain from which $h f q$ had been deleted $(\Delta h f q)$. The red colour afforded to the bacteria by anthraquinone production makes the strain especially suitable for transposon mutagenesis when screening for mutants defective in SM biosynthesis. We screened approximately 60,000 clones for obvious phenotypic alterations. Several mutants were defective in some facets of SM production and showed growth defects (Supplementary Note 3, Extended Data Fig. 3 and Supplementary Table 6); however, only one displayed the desired phenotype. Re-sequencing of this strain, followed by read mapping revealed that the transposon was inserted within an intergenic region associated with the $\operatorname{arc} Z$ sRNA gene (Supplementary Fig. 1).

ArcZ is a well-known Hfq-associated sRNA, which was also present in our list of Hfq-bound sRNAs in P. laumondii (Fig. 1b and Supplementary Table 4). To verify that the observed phenotype was derived from the transposon insertion, we generated a $\triangle \operatorname{arc} Z$ mutant by deleting the major part of the sRNA (Supplementary Fig. 1) and a complemented strain by reintroducing an intact version of $\operatorname{arc} Z$ at the original locus. Northern blot analysis was performed to verify the absence of ArcZ in the deletion mutant and the presence of ArcZ in the WT and the complementation mutant (Fig. 2a). Sequencing of the $\Delta \operatorname{arcZ}$-mutant RNA showed severe transcriptomic changes

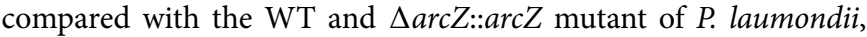
reminiscent of that seen in $P$. laumondii $\Delta h f q$ (Supplementary Note 4, Supplementary Tables 7,8 and Supplementary Fig. 2). The SM production titres in the $\Delta \operatorname{arc} Z$ strain were strongly decreased, similar to that seen in the transposon-insertion mutant, and the complementation strain restored SM production (Fig. $2 \mathrm{c}-\mathrm{h}$ ).

To corroborate the role of ArcZ in SM production, the ArcZ mRNA targets were predicted using CopraRNA ${ }^{23}$ (Supplementary Tables 9 and 10). One hit warranting further investigation was hexA ( $\operatorname{lrh} A)$, which was previously identified as a gene that is highly upregulated in our strains and represses SM production in both P. laumondii ${ }^{24}$ and Xenorhabdus ${ }^{3}$. CopraRNA predicted a 9-bp-long RNA duplex involving the $5^{\prime}$ UTR of hexA and the processed isoform of ArcZ (Fig. 3a). This base-pairing is reminiscent of previously reported ArcZ targets in other bacteria requiring the RNase E-mediated release of the seed region of the sRNA ${ }^{12}$. We also identified a corresponding enriched RNA sequence upstream of the hexA coding sequence at an $\mathrm{OD}_{600}$ of 0.5 in the RIP-seq experiments (Fig. $1 \mathrm{c}$ and Supplementary Fig. 3). We hypothesized that, through Hfq, ArcZ might bind to the hexA transcript leading to repression of HexA, and that Hfq and ArcZ prevent HexA production in laboratory cultures in which SMs are produced, thereby allowing the strain to synthesize SM. However, we expected that hexA would no longer be repressed if either $h f q$ or $\operatorname{arc} Z$ were deleted, resulting in severely reduced SM production. To test this idea, we altered the predicted site of the ArcZ-hexA interaction to a PacI restriction site (TTAATTAA) and created a knock-in of hexA with the modified sequence in a $\Delta$ hexA strain (Supplementary Fig. 4a,b). We predicted that the knock-in of hexA with an altered $5^{\prime}$ UTR would result in a failure of $\mathrm{ArCZ}$ to bind, leading to a reduced SM titre. The SM-production titres of the knock-in mutant with the altered binding site upstream of hexA were indeed greatly reduced (Fig. $2 \mathrm{c}-\mathrm{h}$ ).

To verify the proposed interaction region, we conducted a compensatory base-mutation study in E. coli. The fifth base pair of the proposed interaction region was exchanged in the $\operatorname{arc} Z$ sequence, the hexA 5' UTR or both using site-directed mutagenesis (Fig. 3a). The hexA 5' UTR sequence was fused to the green fluorescent protein $(g f p)$ gene. The GFP output was measured to determine the efficiency of inhibition (Fig. 3b,c). For the control, the GFP signal derived from the expression of hexA::gfp was measured and set to one. When $\mathrm{p}$-arc $Z$ was expressed together with hexA::gfp, HexA repression was increased 32 -fold compared with the control. In addition, when $\mathrm{p}-\operatorname{arc} Z^{\star}$ (G79C) was expressed, ArcZ* was no longer able to repress HexA. HexA repression was only slightly increased compared with the control for hexA*::gfp (C46G) in combination with the native ArcZ, suggesting that ArcZ can still bind to the $5^{\prime}$ UTR of hexA but with substantially reduced efficiency. When $\mathrm{p}-\operatorname{arcZ}^{*}$ (G79C) was combined with hexA*::gfp (C46G), HexA::GFP repression was increased 39 -fold, which confirms our hypothesis that ArcZ binds to the 5' UTR of hexA to repress HexA production. Notably, this base-pairing sequence is located approximately 50 nucleotides upstream of the hexA translational start site (Fig. 3a) and thus ArcZ binding is unlikely to compete with recognition of the mRNA by $30 \mathrm{~S}$ ribosomes ${ }^{25}$. Instead, alignment of the P. laumondii hexA 5' UTR revealed that the ArcZ binding site is CA-rich and highly conserved among other SM-producing bacteria (Supplementary Fig. 5). CA-rich sequences located in proximity to translation initiation sites are well-known translational enhancers and sequestration of these regulatory elements by sRAs has been reported to downregulate gene expression ${ }^{26,27}$, which might also be relevant for the ArcZ-hexA interaction reported here. In addition, we conducted a proteomic analysis with the wild-type (WT), $\Delta \operatorname{arc} Z, \Delta h f q$ and $\Delta h e x A:: h e x A \_P a c I+U T R$ strains of P. laumondii. We used label-free quantification of quadruplicate samples to determine the HexA abundancy in each strain. The levels of HexA were substantially elevated in all mutant strains (11.8- to 22.7-fold; Supplementary Table 11) compared with the WT, further supporting this mechanism of regulation for SM production.

The $\operatorname{arc} Z$ gene and its genomic organization are highly conserved among enterobacterial species ${ }^{10}$ (Fig. 1a). As the control of SMs in Photorhabdus relays a fundamental ability for these bacteria to occupy their specific niche, we investigated the possibility that the same mechanism occurs in the closely related Xenorhabdus. Given the SM reduction in X. szentirmaii $\Delta h f q$, we constructed a $\triangle \operatorname{arcZ}$ mutant in $X$. szentirmaii in a similar fashion to $P$. laumondii by deleting $90 \mathrm{bp}$ of the predicted $\operatorname{arc} Z$ sequence. Using northern blots, we verified that $A r c Z$ was no longer produced by the deletion mutant and that complementation of the deletion led to resumed production of ArcZ (Fig. 3d). We subsequently investigated the transcriptome and SM profile of the WT as well as the deletion and complementation mutants (Fig. 3e and Supplementary Table 12). Consistent with $P$. laumondii, deletion of $\operatorname{arc} Z$ resulted in a global effect on the transcriptome as well as severe reductions in the SM titre, both of which were complemented in the $\triangle \operatorname{arcZ}:$ : $\operatorname{arc} Z$ complementation mutant (Figs. 3e and 4).

Our results highlight the critical role of ArcZ in regulating specialized metabolism in these strains. In fact, the critical nature of the SMs from Photorhabdus and Xenorhabdus in modulating the insect immune response indicated that ArcZ might be required for niche occupation by these bacteria. We observed an inability of the P. laumondii $\triangle \operatorname{arcZ}$ strain to support nematode development (Extended Data Fig. 4), consistent with our earlier observations for the $\Delta h f q$ mutant $^{4}$. However, the same was not seen with X. szentirmaii. 

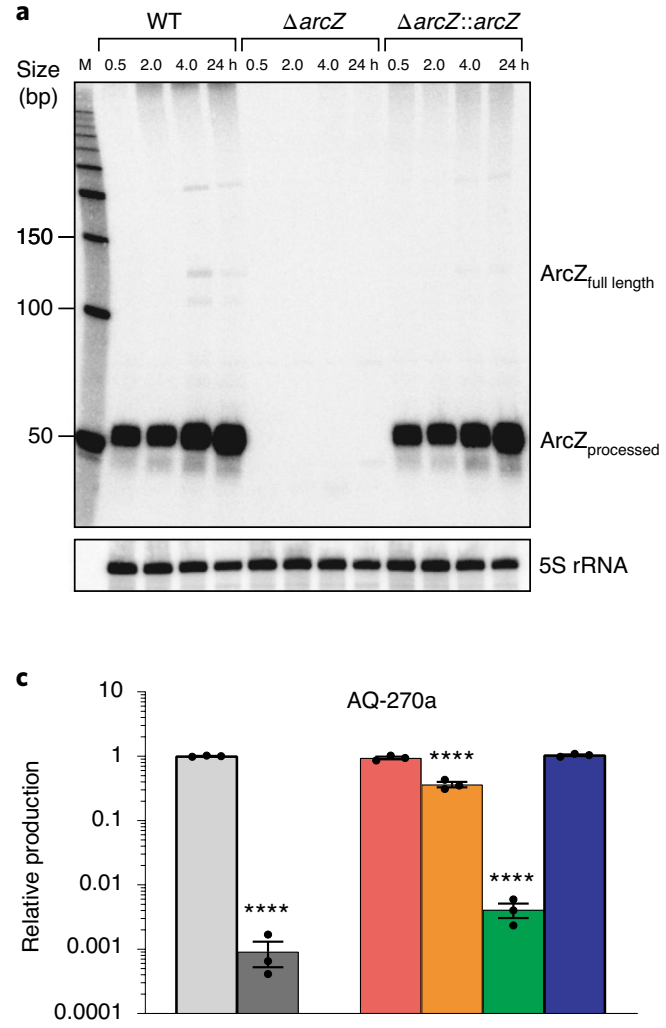

e
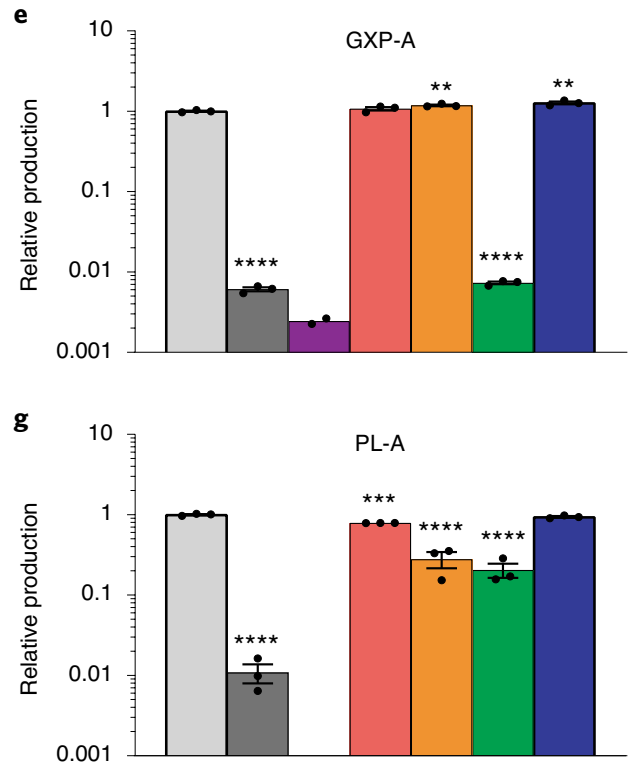
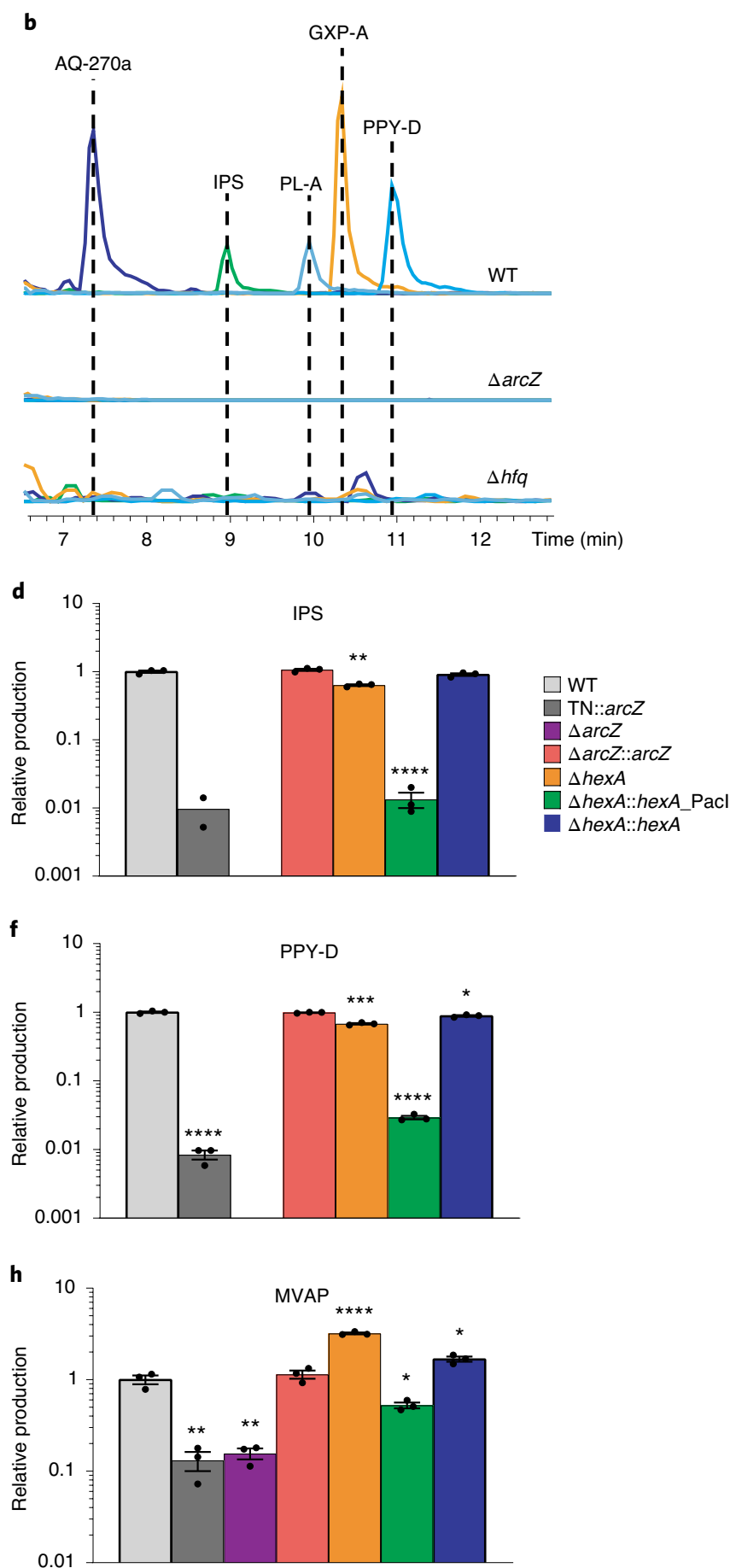

Fig. 2 | Specialized metabolite production in P. laumondii mutant strains. a, ArcZ expression in P. laumondii WT, $\triangle \operatorname{arc} Z$ and $\triangle \operatorname{arcZZ:arcZ~cells,~determined~}$ using northern blot analysis. Total RNA samples were collected at three different $\mathrm{OD}_{600}$ values $(0.5,2$ and 4$)$ and after $24 \mathrm{~h}$ of growth. Probing for $5 \mathrm{~S}$ ribosomal RNA served as a loading control. The blot image is representative of two biologically independent replicates. $M$, molecular weight markers. b, Comparison of the relative production titres of the major SMs produced by the $P$. laumondii WT, $\Delta$ arc $Z$ and $\Delta h f q$ strains. The extracted ion chromatograms of anthraquinone (AQ-270a), isopropylstilbene (IPS), phurealipid A (PL-A), GXP-A and photopyrone D (PPY-D) in the WT and mutant strains are depicted. c-h, HPLC-MS/MS quantification of AQ-270a (c), IPS (d), GXP-A (e), PPY-D (f), PL-A (g) and mevalagmapeptide (MVAP; $\mathbf{h}$ ) in P. laumondii WT, TN::arcZ, $\triangle \operatorname{arcZ}, \triangle \operatorname{arcZ}:: \operatorname{arc} Z, \triangle$ hexA, $\triangle$ hexA::hexA_Pacl and $\triangle$ hexA::hexA. All bars represent the relative production in comparison to the WT. Data are presented as the mean \pm s.e.m. The dots represent biologically independent replicates $(n=3) .{ }^{\star} P<0.05$, ${ }^{\star \star} P<0.005$, ${ }^{\star \star \star} P<0.0005$ and ${ }^{\star \star \star \star} P<0.0001$. Statistical significance of the relative production compared with the WT production levels was calculated using a two-sided unpaired $t$-test. The exact $P$ values were as follows (from left to right): $\mathbf{c},<0.0001$, not determined (n.d.), $0.2,<0.0001,<0.0001$ and 0.24 . d, n.d., n.d., $0.24,0.0005,<0.0001$ and 0.12 . e, $<0.0001$, n.d., $0.18,0.0018,<0.0001$ and 0.0037 . f, $<0.0001$, n.d., $0.56,0.0003,<0.0001$ and 0.013 . g, $<0.0001$, n.d., $0.0001,<0.0001,<0.0001$ and $0.059 . \mathbf{h}, 0.0006,0.0008,0.34,<0.0001,0.011$ and 0.0058 . Details of all analysed compounds can be found in Supplementary Table 16. 

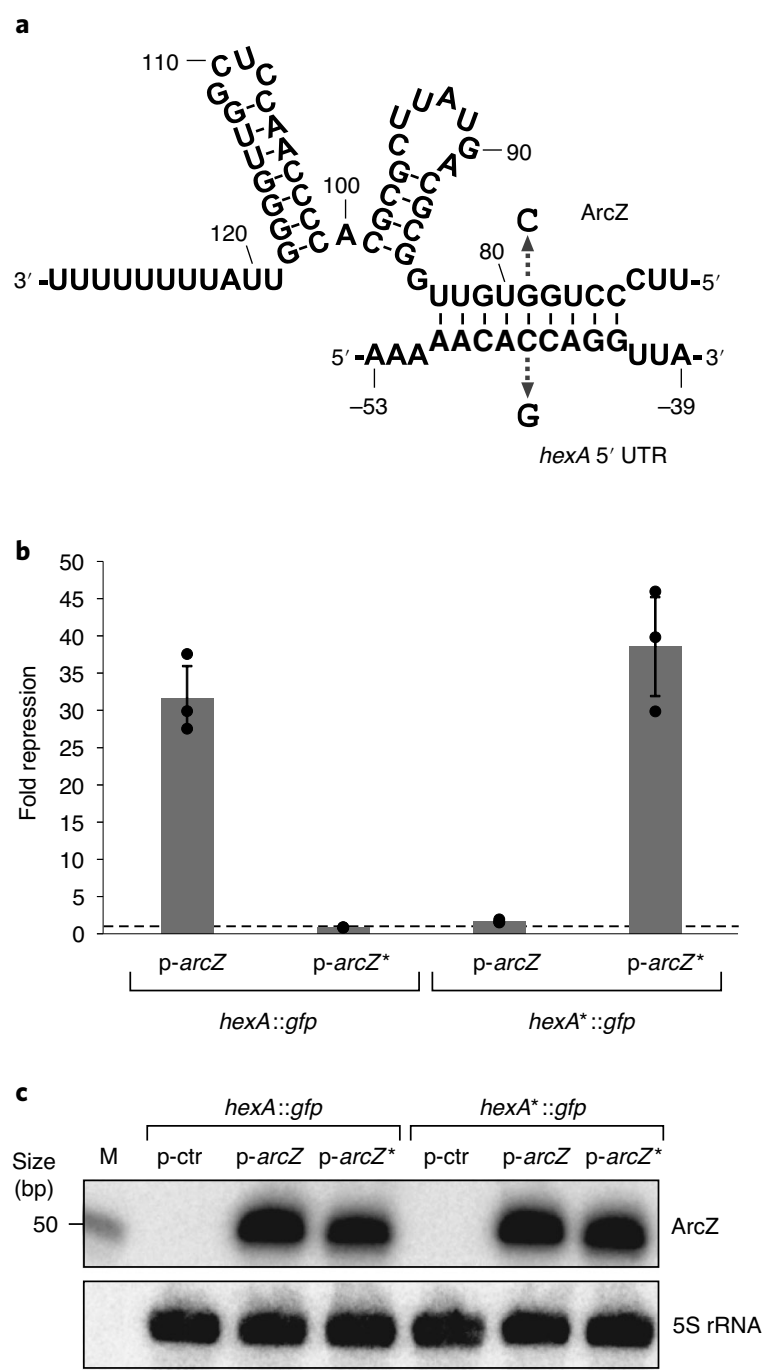

d

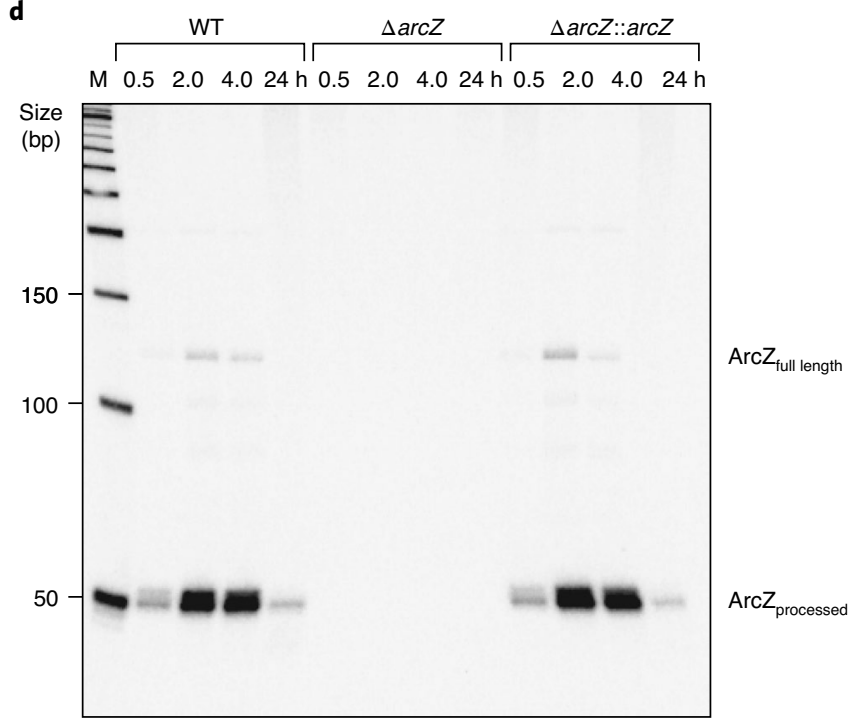

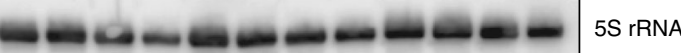

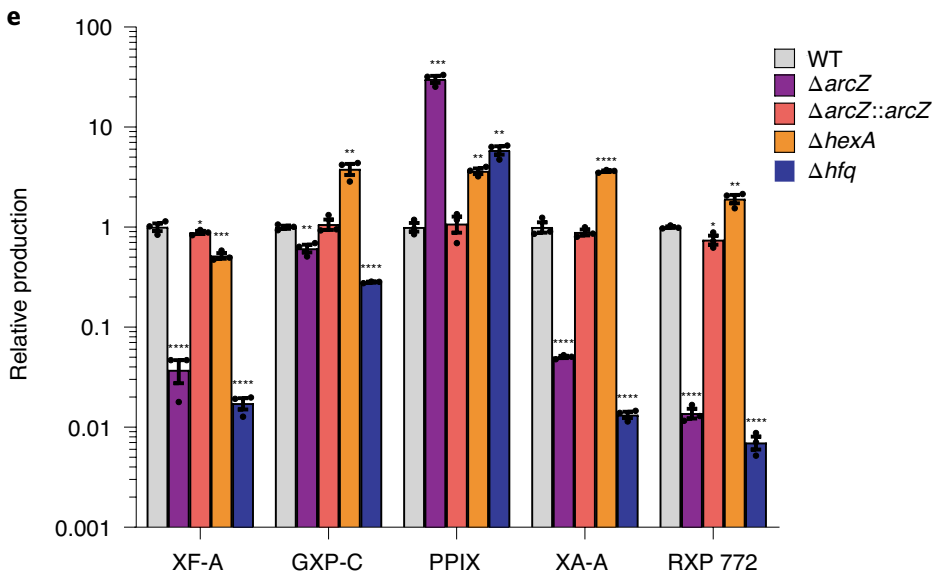

Fig. 3 | Base-pairing between ArcZ and hexA. a, Predicted base-pairing interaction of ArcZ with the 5' UTR of the hexA mRNA. The arrows indicate the single nucleotide mutations tested in b. b. GFP signals derived from co-expression of a plasmid harbouring the $5^{\prime}$ UTR of hexA fused to gfp (hexA::gfp) or the same fusion with a single point mutation (C46G; hex $A^{\star}:: g f p$ ) with the control (p-ctr), p-arcZ or p-arcZ* (G79C) plasmids. The levels of GFP of the strains carrying $p$-ctr were set to one (dashed line). Data are presented as the mean \pm s.d. The dots represent biologically independent replicates ( $n=3$ ). $\mathbf{c}$, Northern blot analysis of ArcZ expression corresponding to the GFP expression assay shown in b. d, ArcZ expression in X. szentirmaii WT, $\Delta$ arcZ and $\Delta$ arcZ::arcZ cells, determined using northern blot analysis. Total RNA samples were collected at three different $O_{600}$ values $(0.5,2$ and 4$)$ and after $24 \mathrm{~h}$ of growth.

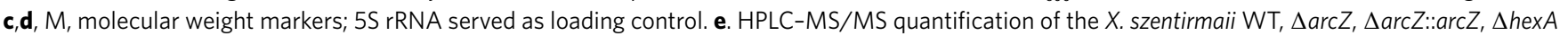
and $\Delta h f q$ strains. The relative production of xenofuranone A (XF-A), GXP-C, PPIX, xenoamicin A (XA-A) and rhabdopeptide 772 (RXP 772 ) are shown. See also Supplementary Table 16. ${ }^{\star} P<0.05,{ }^{\star \star} P<0.005,{ }^{\star \star \star} P<0.0005$ and ${ }^{\star * \star *} P<0.0001$; statistical significance of the relative production compared with WT production levels calculated using a two-sided unpaired $t$-test. The exact $P$ values were as follows (from left to right): XF- $A,<0.0001,0.021,0.0001$ and <0.0001; GXP-C, 0.0034, 0.059, 0.0021 and <0.0001; PPIX, 0.0001, 0.8, 0.0008 and 0.0007; XA-A, <0.0001, 0.14, <0.0001 and <0.0001; and RXP $772,<0.0001,0.02,0.0041$ and $<0.0001$. Details of all of the analysed compounds can be found in Supplementary Table 16. Data are presented as the mean \pm s.e.m. The dots represent biologically independent replicates $(n=3)$.

We suspect this might be because of the observed increase in protoporphyrin IX (PPIX) production in the X. szentirmaii $\Delta$ arcZ strain (Supplementary Note 5). PPIX is a precursor of haem, which is an important cofactor for key biological processes such as oxidative metabolism ${ }^{28}$, protein translation ${ }^{29}$, maintenance of protein stability $^{30}$ and many others. However, PPIX cannot be synthesized de novo by Caenorhabditis elegans and other nematodes ${ }^{31}$. The nematodes therefore rely on external PPIX sources (such as from symbiotic bacteria), which positively affects their growth, reproduction and development ${ }^{32}$. It is interesting that, despite P. laumondii also being capable of producing PPIX, reproduction of the Heterorhabditis nematode was not supported in the $\Delta \operatorname{arcZ}$ mutant or $\Delta h f q$ strain. This is possibly indicative of the nematode-specific requirements for reproduction, which may also include isopropylstilbene as an essential factor in Heterorhabditis ${ }^{17}$, where no analogous compound is known to be required for Steinernema.

Nearly all of the SM-related genes analysed in both Xenorhabdus and Photorhabdus were found to be downregulated in the $\triangle \operatorname{arcZ}$ mutant, in accordance with impaired SM production (Fig. 4a,b). This provides a chemical background that is devoid of natural products, which allows for the isolation and identification of a desired compound due to the absence of compounds with similar retention 


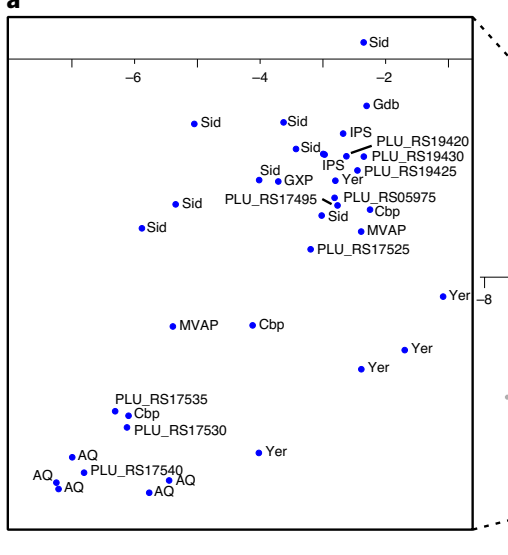

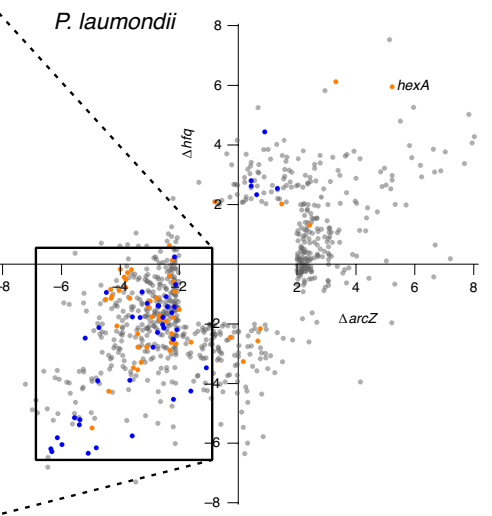

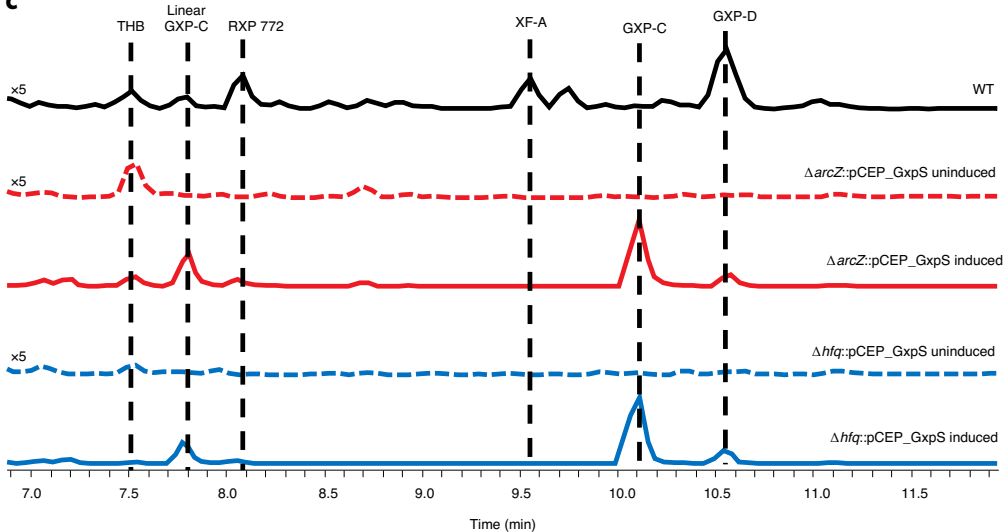

b

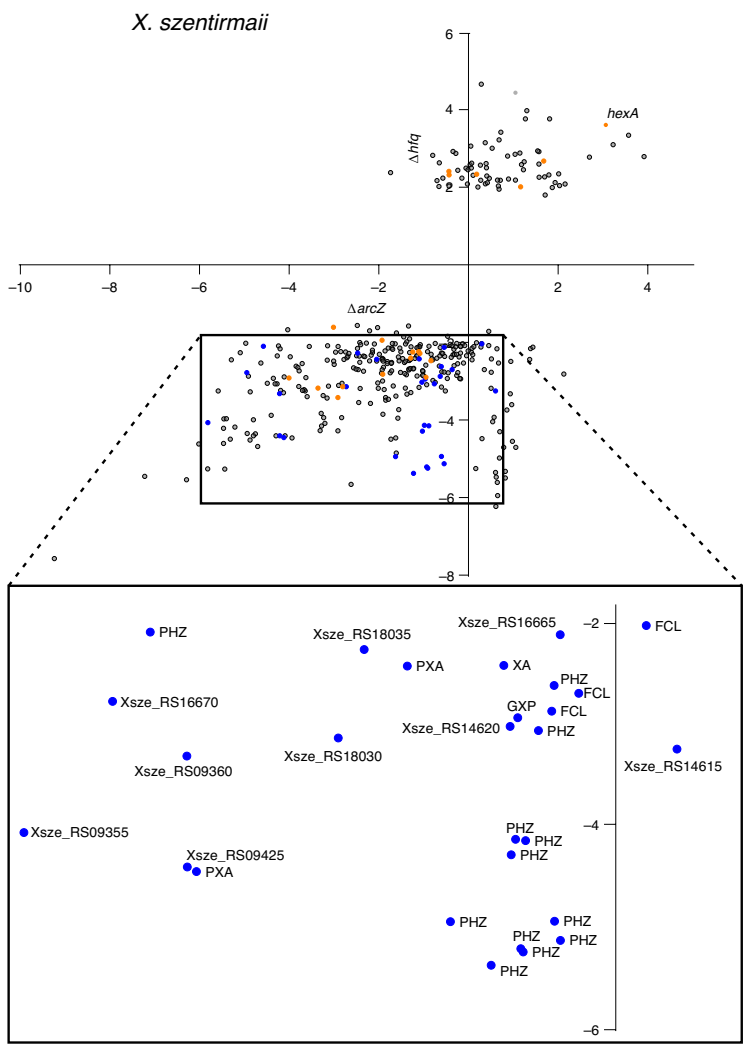

Fig. 4 | Secondary metabolites are regulated by arcZ and $\boldsymbol{h f q}$ regulons. a,b, Comparison of the Arc $Z$ and Hfa regulons in $P$. laumondii (a) and $X$. szentirmaii (b). The scatterplots show individual coding sequences and their corresponding regulatory changes compared with the WT in either the $\Delta$ arc $Z$ ( $x$ axis) or $\Delta h f q$ ( $y$ axis) mutants, with the SMs (blue dots) and regulators (orange dots) highlighted. Inset: only SM-related coding sequences are shown, including those associated with anthraquinone (AQ), mevalagmapeptide (MVAP), carbapenem (Cbp), yersiniabactin (Yer), GXP, siderophore (Sid), isopropylstilbene (IPS), glidobactin (Gdb), phenazine (PHZ), fabclavine ( $F C L)$, xenoamicin (XA) and pyrrolizixenamide (PXA). c, Base peak chromatograms of $X$. szentirmaii WT, $\Delta$ arcZ::pCEP_GxpS (uninduced and induced) and $\Delta h f q:: p C E P \_G x p S$ (uninduced and induced) strains. Peaks correspond to (cyclo)tetrahydroxybutyrate $(\mathrm{THB})^{58}$, linear GXP-C, rhabdopeptide 772 (RXP 772), xenofuranone A (XF-A), as well as cyclic GXP-C and GXP-D. A ×5 zoom was applied to the base peak chromatograms in the uninduced and WT samples.

times. Therefore, $\triangle \operatorname{arc} Z$ mutants could offer a powerful tool for the (over-)production and identification of previously undescribed natural products. As a proof of concept, we conducted a promotor exchange in front of gxpS in both $X$. szentirmaii $\triangle a r c Z$ and $X$. szentirmaii $\Delta h f q$, and compared GameXPeptide (GXP)-C production after induction to the WT (Fig. 4c). The production of GXP-C was found to be increased 90.4 -fold $( \pm 4.7)$ in X. szentirmaii $\triangle \operatorname{arcZ}:$ :pCEP_GxpS and increased 138.6-fold $( \pm 17.1)$ in X. szentirmaii $\Delta h f q::$ CEP_GxpS compared with the WT (Fig. 4c). The striking increase in production as well as the dramatically reduced chemical background in both strains highlights the potential for exploiting this regulatory cascade for selective SM production in a strain that is well-suited for natural product detection. We recently showed that this strategy could be applied in a high-throughput manner for rapid screening of bioactivities ${ }^{33}$. The same strategy used here in a $\Delta \operatorname{arc} Z$ strain demonstrates an alternative route to activation, without the complex perturbations associated with deleting the major RNA chaperone in these bacteria. Interestingly, some comparisons between these mechanisms can be drawn in other SM-producing Enterobacteriaceae (Fig. 1a). Erwinia is a genus of plant-pathogenic bacteria that produce SMs, where Hfq and ArcZ have both been implicated in virulence ${ }^{34}$, whereas HexA is a negative regulator of secondary metabolites in these bacteria ${ }^{35}$. Similar parallels can also be seen in Serratia ${ }^{36-38}$ and Pseudomonas ${ }^{39}$, two other prolific SM producers. Although further investigations will be required to ascertain whether these apparent similarities represent identical mechanisms, the conserved nature of ArcZ in other SM-producing Enterobacteriaceae could suggest that this strategy may yield fresh avenues for rapid investigation into SM biosynthesis in other taxa.

\section{Methods}

Bacterial culture conditions. All Photorhabdus and Xenorhabdus strains were cultured in Luria-Bertani (LB) medium for at least $16 \mathrm{~h}$ with shaking at $30^{\circ} \mathrm{C}$. The E. coli strains were cultured in LB for at least $16 \mathrm{~h}$ with shaking at $37^{\circ} \mathrm{C}$. The medium was supplemented with chloramphenicol $\left(34 \mu \mathrm{g} \mathrm{ml}^{-1}\right)$, ampicillin $\left(100 \mu \mathrm{g} \mathrm{ml}^{-1}\right)$, rifampicin $\left(50 \mu \mathrm{g} \mathrm{ml}^{-1}\right)$ or kanamycin $\left(50 \mu \mathrm{g} \mathrm{ml}^{-1}\right)$ when appropriate. Promotor-exchange mutants were induced by adding $\mathrm{L}$-arabinose $(2 \%, \mathrm{vol} / \mathrm{vol})$ to the cultures. All of the plasmids and strains used in this study are listed in Supplementary Tables 13 and 14 .

Nematode bioassays. All nematodes were cultivated in Galleria mellonella and collected on white traps as previously described ${ }^{40}$. The nematode bioassays were also performed as described elsewhere ${ }^{4}$.

Creation of a transposon-mutant library. For the transposon mutagenesis, the plasmid pSAM_Kan (containing the mariner transposon) was constructed using pSAM_BT ${ }^{41}$ as a template. To do this, the plasmid was linearized using the primers NN191 and NN192. The kanamycin-resistance cassette was amplified from the pCOLA_ara_tacI plasmid using the primers NN193 and NN194, introducing complementary overhangs to PSAM_BT at both ends of the PCR fragment. 
The kanamycin-resistance cassette was fused with the linearized pSAM_BT plasmid using Hot Fusion cloning, thereby replacing the erythromycin-resistance cassette with kanamycin resistance. E. coli ST18 was transformed with the plasmid pSAM_Kan and further used for the creation of the transposon-mutant library of $P$. laumondii TTO1 through conjugation. Transposon-insertion mutants were selected on LB agar containing kanamycin. All primer sequences are listed in Supplementary Table 15.

Construction of mutant strains. For the deletion of the majority of ArcZ in P. laumondii TTO1, a 1,123-bp upstream and a 1,014-bp downstream product were amplified using the primers NN276 and NN277, and NN278 and NN279, respectively. The PCR products were fused using the complementary overhangs introduced by the primers and cloned into the pEB17 plasmid linearized with PstI and BglII. The resulting plasmid was used for transformation of E. coli s17-1 $\lambda$ pir. Conjugation of the plasmid in the P. laumondii strains and generation of deletion strains by homologous recombination through counter selection was done as previously described ${ }^{42}$. The deletion mutants were verified by PCR using the primers NN281 and NN282, which yielded a 632-bp fragment for mutants genetically equal to the WT and a 502-bp fragment for the desired deletion mutant. Complementation of the ArcZ deletion was achieved by inserting the full and intact version of ArcZ at the original locus. To do this, a 2,207-bp PCR product including the upstream and downstream region required for homologous recombination and the full-length ArcZ was amplified using the primers NN276 and NN279. The fragment was cloned into pEB17 as described earlier. The verified plasmid construct was used to transform E. coli s17-1 $\lambda$ pir cells. The plasmid was transferred into P. laumondii $\Delta a r c Z$ by conjugation and integrated into the genome of $P$. laumondii $\triangle \operatorname{arc} Z$ by homologous recombination. The knock-in mutant was generated by a second homologous recombination through counter selection on LB plates containing 6\% sucrose. Knock-in mutants were verified by PCR using the primers NN281 and NN282, yielding a 632-bp fragment. The same strategy was used for the construction of the mutant strains in X. szentirmaii. To generate the promotor-exchange mutants in front of $\operatorname{gxp} S$, the plasmid pCEPKMR_ORF00346 was transferred into $X$. szentirmaii $\Delta$ arcZ and $X$. szentirmaii $\Delta h f q$ by conjugation and integrated into the genome by homologous recombination.

DNA extraction. Genomic DNA was extracted using the Gentra Puregene yeast/ bact. kit (Qiagen) following the manufacturer's instructions. For sequencing of transposon-insertion mutants, genomic DNA was extracted using the DNeasy blood and tissue kit (Qiagen).

DNA sequencing and identification of the transposon-insertion site. DNA isolated from the transposon-insertion mutants was sequenced on an Illumina NextSeq platform. DNA libraries were constructed using the Nextera XT DNA preparation kit (Illumina) and whole-genome sequencing was performed using $2 \times 150$ bp paired-end chemistry. A sequencing depth of $>50 \times$ was targeted for each sample. Genomes were assembled using SPAdes (v. 3.10.1) ${ }^{43}$ and annotated using Prokka v. 1.12 (ref. ${ }^{44}$ ). The completed genome sequences were analysed and viewed using Geneious v. 6 and 9.1 (https://www.geneious.com).

RNA extraction, sequencing and analysis. Pre-cultures of $P$. laumondii TTO1, $X$. szentirmaii DSM16338 and their respective ArcZ deletion and knock-in mutants were cultured overnight in LB broth with shaking at $30^{\circ} \mathrm{C}$. The pre-cultures were used the following day to inoculate fresh $\mathrm{LB}$ at an $\mathrm{OD}_{600}$ of 0.3 . The cells were cultured to the mid-exponential phase (the OD values for each experiment can be found in Supplementary Table 3). RNA was extracted using an RNeasy mini kit (Qiagen) following the manufacturer's instructions. To facilitate cell lysis, the cells were pelleted and snap-frozen in liquid nitrogen for $1 \mathrm{~min}$ after removing the supernatant. After thawing and resuspending in lysis buffer, the cells were vortexed for $30 \mathrm{~s}$ before proceeding with the protocol. The RNA for the sRNA libraries were extracted in duplicate during the mid-exponential phase for P. laumondii TTO1 and $X$. szentirmaii.

The RNA was sequenced through $150 \mathrm{bp}$ paired-end sequencing by Novogene following rRNA depletion with a RiboZero kit and library preparation following the Illumina protocol for strand-specific libraries. The raw data were trimmed using Trimmomatic ${ }^{45}$ and mapped to the reference genome downloaded from NCBI (NC_005126.1 for P. laumondii and NZ_NIBV00000000.1 for X. szentirmaii) using bowtie 2 (v2.3.4.3 ${ }^{46}$. The resulting .sam files were converted to bam files using samtools (v1.8) $)^{47}$ and featureCounts (a part of the subread package) ${ }^{48}$ was used to count reads mapping to annotated genes. The count files were then uploaded to degust (http://degust.erc.monash.edu/) and analysed using the voom/ limma method of normalization. Only genes with an absolute fold change $>2$ and false discovery rate $<0.01$ were considered significantly regulated. Statistical analysis was performed in $\mathrm{R}$ (v. 3.6.1) on the degust platform, where the exact code is available to view.

Northern blot analysis. For the northern blot analyses, total RNA was prepared and analysed as described previously ${ }^{49}$. Briefly, the RNA samples were separated on $6 \%$ polyacrylamide and $7 \mathrm{M}$ urea gels and transferred to Hybond-XL membranes (GE Healthcare) by electro-blotting. The membranes were hybridized in
Roti-Hybri-Quick buffer (Roth) at $42^{\circ} \mathrm{C}$ with gene-specific $\left[{ }^{32} \mathrm{P}\right]$ end-labelled DNA oligonucleotides, and washed in three subsequent steps with $5 \times, 1 \times$ and $0.5 \times$ SSC (in $0.1 \%$ SDS) wash buffer. Signals were visualized on a Typhoon FLA 7000 phosphorimager (FUJIFILM). The oligonucleotides used for the northern blot analyses are listed in Supplementary Table 15.

Compensatory base mutation and GFP fluorescence assay. The plasmids pMH078 and pMH079 were generated using Gibson assembly ${ }^{50}$. For plasmid pMH078, the $\operatorname{arcZ}$ gene was amplified using P. laumondii TTO1 genomic DNA with the oligonucleotides KPO-6147 and KPO-6148, and fused into a pEVS143 vector backbone ${ }^{51}$, linearized with KPO-0092 and KPO-1397. To construct the plasmid pMH079, the 5' UTR and the first 20 amino acids of hexA were amplified using P. laumondii TTO1 genomic DNA with KPO-6145 and KPO-6146, and the pXG10- $g f p$ vector ${ }^{52}$ was linearized with KPO-1702 and KPO-1703. The plasmids pMH078 and pMH079 served as templates to insert single point mutations in the $\operatorname{arcZ}$ gene as well as the hexA 5' UTR using site-directed mutagenesis and the oligonucleotide combinations KPO-6156 and KPO-6157, and KPO-6164 and KPO-6165, respectively, yielding the plasmids pMH080 and pMH081.

Target regulation using GFP reporter fusions was analysed as described previously ${ }^{52}$. E. coli Top 10 cells were cultured overnight in $\mathrm{LB}$ medium $\left(37^{\circ} \mathrm{C}\right.$ with shaking at 200 r.p.m.). Three independent cultures were used for each strain. The cells were washed in PBS and the GFP fluorescence intensity was determined using a Spark $10 \mathrm{M}$ plate reader (TECAN). Samples that did not express fluorescence proteins were used as controls to subtract the background fluorescence.

Cappable-seq analysis. The Cappable-seq was performed as previously described by Vertis Biotechnologies. The raw sequences were trimmed using Trimmomatic ${ }^{45}$ and mapped using bowtie2 (ref. ${ }^{46}$ ) to NC 005126.1 for P. laumondii and NZ_NIBV00000000.1 for X. szentirmaii. The transcriptional start sites were detected using the built-in TSS detection function read of Xplorer (v2.2.3) ${ }^{54}$ with the following settings: use only single perfect matches, minimum number of read starts $=100$, minimum percent coverage increase $=750$, detect previously undescribed transcripts, minimum transcript extension $=40$, maximum distance to feature of leaderless transcripts $=5$ and associate neighbouring TSS within $3 \mathrm{bp}$.

RIP-seq analysis. Overnight cultures of $P$. laumondii TTO1 (WT and $\mathrm{Hfq}^{3 \times \mathrm{FLAG}}$ ) were inoculated into fresh $\mathrm{LB}$ medium in duplicate and cultured at $30^{\circ} \mathrm{C}$ with shaking at 200 r.p.m. The bacteria were harvested by centrifugation at 4,000 r.p.m. for $15 \mathrm{~min}$ at $4{ }^{\circ} \mathrm{C}$ when the cells reached $\mathrm{OD}_{600}=0.5$ and $\mathrm{OD}_{600}=5.0$. The cell pellets were resuspended in $1 \mathrm{ml}$ lysis buffer $(20 \mathrm{mM}$ Tris $\mathrm{pH} 8.0$, $150 \mathrm{mM} \mathrm{KCl}, 1 \mathrm{mM} \mathrm{MgCl}$ and $1 \mathrm{mM}$ dithiothreitol) and pelleted again by centrifugation $\left(5 \mathrm{~min}, 11,200 \mathrm{~g}, 4^{\circ} \mathrm{C}\right)$. The supernatants were discarded and the pellets were snap-frozen in liquid nitrogen. After thawing on ice, the cells were resuspended in $800 \mu \mathrm{l}$ lysis buffer and transferred into tubes containing $300 \mu \mathrm{l}$ glass beads to break the cells using a Bead Ruptor $(2 \times 150 \mathrm{~s}$, with a 2 min break on ice in between). After a short centrifugation $\left(15,000 \mathrm{~g}, 4^{\circ} \mathrm{C}\right)$, the lysates were transferred into fresh pre-cooled tubes and centrifuged for $30 \mathrm{~min}$ at $15,200 \mathrm{~g}$ at $4{ }^{\circ} \mathrm{C}$. The cleared lysates were transferred into fresh tubes and incubated with $35 \mu$ l FLAG-antibody (monoclonal anti-FLAG M2; Sigma, F1804) with rotation for $45 \mathrm{~min}$ at $4^{\circ} \mathrm{C}$, followed by the addition of $75 \mu$ l Protein G Sepharose (Sigma, P3296) and rotation for $45 \mathrm{~min}$ at $4^{\circ} \mathrm{C}$ again. After five wash steps with lysis buffer (by inverting the tube gently and centrifuging for $4 \mathrm{~min}$ at $4{ }^{\circ} \mathrm{C}$ ), the samples were subjected to RNA and protein separation using phen ol:chloroform:isoamylalcohol (25:24:1, pH 4.5; Roth) extraction. The upper phase (approximately $500 \mu \mathrm{l}$ ) was transferred into a fresh tube and precipitated overnight at $-20^{\circ} \mathrm{C}$ with $1.5 \mathrm{ml} \mathrm{EtOH}: \mathrm{Na}$ (acetate) $(30: 1)$ and $1.5 \mu \mathrm{l} \mathrm{GlycoBlue}$ (Ambion, AM9516). After centrifugation at 11,200 r.p.m. for $30 \mathrm{~min}$ at $4{ }^{\circ} \mathrm{C}$, the RNA pellets were washed with $500 \mu \mathrm{l} 70 \% \mathrm{EtOH}$, dried and resuspended in $15.5 \mu \mathrm{l}$ nuclease-free $\mathrm{H}_{2} \mathrm{O}$. The RNA was treated with $2 \mu \mathrm{l}$ DNase I, $0.5 \mu$ l RNase inhibitor and $2 \mu \mathrm{l} 10 \times \mathrm{DNase}$ buffer at $37^{\circ} \mathrm{C}$ for $30 \mathrm{~min}$. The samples were then supplemented with $100 \mu \mathrm{H}_{2} \mathrm{O}$ and again subjected to a phenol:chloroform:isoam ylalcohol extraction. The upper phase (approximately $120 \mu \mathrm{l}$ ) was transferred to a fresh tube with the addition of 2.5-3 volumes (about $350 \mu \mathrm{l}$ ) of EtOH:Na(acetate) (30:1) and stored at $-20^{\circ} \mathrm{C}$ overnight for RNA precipitation. The RNA pellets were harvested via centrifugation at 13,000 r.p.m. for $30 \mathrm{~min}$ at $4^{\circ} \mathrm{C}$, and washed with $500 \mu \mathrm{l}$ of $70 \% \mathrm{EtOH}$, dried and resuspended in nuclease-free $\mathrm{H}_{2} \mathrm{O}$. Complementary DNA libraries were prepared using a NEBNext small RNA library prep set for Illumina (NEB, E7300S) according to the manufacturer's instructions and sequenced on a HiSeq 1500 system in single-read mode with a read length of 100 nucleotides.

For RIP-seq analysis, the enriched-control sample pairs were normalized to the number of raw reads present after trimming. Depth counts of all samples were obtained using samtools (v1.8) ${ }^{47}$. Only nucleotide positions with a depth of at least 50 reads in the enriched samples were taken for further analysis. The corresponding depth in the unenriched samples was matched for each nucleotide. A region was considered to be enriched if the enrichment factor was at least three and the corresponding 'enriched' nucleotide was present in both sample pairs. Finally, we considered a region to be enriched if more than five consecutive nucleotides were identified as enriched. 
ArcZ-binding prediction. ArcZ from E. coli was used to define the boundaries of ArcZ in Xenorhabdus and Photorhabdus. We then took our annotated ArcZ sequence together with several ArcZ homologues from other Enterobacteriaceae (listed in Supplementary Table 9) and used the online CopraRNA tool ${ }^{23}$, a part of the Freiburg RNA tools suite ${ }^{55}$, with default parameters.

Metabolite extraction and HPLC-MS/MS analysis. Fresh LB medium (10 ml) was inoculated with an overnight culture to an $\mathrm{OD}_{60}$ of 0.1 . After $72 \mathrm{~h}$ of cultivation at $30^{\circ} \mathrm{C}$ with shaking, $1 \mathrm{ml}$ of the culture was removed from the culture, centrifuged for $20 \mathrm{~min}$ at 13,300 r.p.m. and the supernatant was directly subjected for analysis by HPLC-MS/MS using a Dionex Ultimate 3000 system with a Bruker AmaZon X mass spectrometer. The peak areas of the compounds were quantified using TargetAnalysis 1.3 (Bruker). All of the analysed compounds are listed in Supplementary Table 16.

Proteome analysis. The details of the proteomics procedure were previously published ${ }^{56}$. In short, to extract proteins from $P$. laumondii frozen cell pellets, $300 \mu \mathrm{l}$ lysis buffer $\left(0.5 \% \mathrm{Na}\right.$-desoxycholate in $\left.100 \mathrm{mM} \mathrm{NH}_{4} \mathrm{HCO}_{3}\right)$ was added to the cell pellet and incubated at $95^{\circ} \mathrm{C}$ for $10 \mathrm{~min}$. The protein concentration in the supernatant was determined using a BCA protein assay kit (Thermo Fisher, 23252). Reduction and alkylation was performed at $95^{\circ} \mathrm{C}$ using $5 \mathrm{mM}$ TCEP and $10 \mathrm{mM}$ chloroacetamide for $15 \mathrm{~min}$. The protein $(50 \mu \mathrm{g})$ was transferred to fresh reaction tubes and protein digestion was carried out overnight at $30^{\circ} \mathrm{C}$ with $1 \mu \mathrm{g}$ trypsin (Promega). After the digestion, the peptides were desalted using CHROMABOND spin columns (Macherey-Nagel) that were conditioned with $500 \mu \mathrm{l}$ acetonitrile and equilibrated with $500 \mu \mathrm{l}$ and $150 \mu \mathrm{l} 0.1 \%$ trifluoroacetic acid (TFA). After loading, the peptides were washed with $500 \mu \mathrm{l}$ of $0.1 \%$ TFA in acetonitrile: $\mathrm{H}_{2} \mathrm{O}(5: 95)$ and eluted with $400 \mu \mathrm{l}$ of $0.1 \%$ TFA in acetonitrile: $\mathrm{H}_{2} \mathrm{O}$ (50:50). The peptides were concentrated and dried under vacuum at $50^{\circ} \mathrm{C}$ and dissolved in $100 \mu \mathrm{l}$ of $0.1 \%$ TFA by $25 \mathrm{~s}$ of sonication and incubation at $22^{\circ} \mathrm{C}$ with shaking at 1,200 r.p.m. for $5 \mathrm{~min}$. Peptides $(1 \mu \mathrm{g})$ were analysed using HPLC-MS/MS

The HPLC-MS/MS analysis including label-free quantification was carried out as previously described ${ }^{56}$, with minor modifications.

HPLC-MS/MS analysis of protein digests was performed on a Q-Exactive Plus mass spectrometer connected to an electrospray ion source (Thermo Fisher Scientific). Peptide separation was carried out using an Ultimate 3000 nanoLC-system (Thermo Fisher Scientific) equipped with C18 resin column packed in-house (Magic C18 AQ $2.4 \mu \mathrm{m}$; Dr. Maisch). The peptides were first loaded onto a C18 pre-column (pre-concentration set-up) and then eluted in backflush mode with a gradient from $98 \%$ solvent A $(0.15 \%$ formic acid $)$ and $2 \%$ solvent B (99.85\% acetonitrile and $0.15 \%$ formic acid) to $35 \%$ solvent B over $30 \mathrm{~min}$. Label-free quantification was done using Progenesis QI software (Nonlinear Dynamics, v2.0) and the MS/MS search was performed in MASCOT (v2.5, Matrix Science) against the UniProt P. laumondii protein database. The following search parameters were used: full tryptic search with two missed cleavage sites, $10 \mathrm{ppm}$ MS1 and 0.02 Da fragment ion tolerance. Carbamidomethylation (C) as fixed, and oxidation $(\mathrm{M})$ and deamidation $(\mathrm{N}, \mathrm{Q})$ as variable modifications. The progenesis outputs were further processed using SafeQuant ${ }^{57}$

Reporting Summary. Further information on research design is available in the Nature Research Reporting Summary linked to this article.

\section{Data availability}

All .mzXML files from the HPLC-MS/MS runs are available at MassIVE (https:// massive.ucsd.edu) under the ID MSV000084163. Raw sequence data are available at the European nucleotide archive (https://www.ebi.ac.uk/ena/) under project accession numbers PRJEB33827 and PRJEB24159. The proteomic data can be accessed at PRIDE (https://www.ebi.ac.uk/pride/) with the project accession number PXD019095. Source data are provided with this paper.

Received: 11 August 2019; Accepted: 16 September 2020; Published online: 02 November 2020

\section{References}

1. Stock, S. P., Campbell, J. F. \& Nadler, S. A. Phylogeny of Steinernema travassos, 1927 (Cephalobina: Steinernematidae) inferred from ribosomal DNA sequences and morphological characters. J. Parasitol. 87, 877-889 (2001).

2. Forst, S., Dowds, B., Boemare, N. \& Stackebrandt, E. Xenorhabdus and Photorhabdus spp.: bugs that kill bugs. Annu. Rev. Microbiol. 51, 47-72 (1997).

3. Engel, Y., Windhorst, C., Lu, X., Goodrich-Blair, H. \& Bode, H. B. The global regulators Lrp, LeuO, and HexA control secondary metabolism in entomopathogenic bacteria. Front. Microbiol. 8, 209 (2017).

4. Tobias, N. J. et al. Photorhabdus-nematode symbiosis is dependent on hfq-mediated regulation of secondary metabolites. Environ. Microbiol. 19, 119-129 (2017).
5. Santiago-Frangos, A. \& Woodson, S. A. Hfa chaperone brings speed dating to bacterial sRNA. Wiley Interdiscip. Rev. RNA 9, e1475 (2018).

6. Holmqvist, E. \& Vogel, J. RNA-binding proteins in bacteria. Nat. Rev. Microbiol. 16, 601-615 (2018).

7. Carrier, M.-C., Lalaouna, D. \& Massé, E. Broadening the definition of bacterial small RNAs: characteristics and mechanisms of action. Annu. Rev. Microbiol. 72, 141-161 (2018).

8. Vogel, J. \& Luisi, B. F. Hfq and its constellation of RNA. Nat. Rev. Microbiol. 9, 578-589 (2011).

9. Chao, Y., Papenfort, K., Reinhardt, R., Sharma, C. M. \& Vogel, J. An atlas of Hfq-bound transcripts reveals $3^{\prime}$ UTRs as a genomic reservoir of regulatory small RNAs. EMBO J. 31, 4005-4019 (2012).

10. Papenfort, K. et al. Specific and pleiotropic patterns of mRNA regulation by ArcZ, a conserved, Hfq-dependent small RNA. Mol. Microbiol. 74, 139-158 (2009).

11. Mandin, P. \& Gottesman, S. Integrating anaerobic/aerobic sensing and the general stress response through the ArcZ small RNA. EMBO J. 29, 3094-3107 (2010).

12. Chao, Y. et al. In vivo cleavage map illuminates the central role of RNase E in coding and non-coding RNA pathways. Mol. Cell 65, 39-51 (2017).

13. Beemelmanns, C., Guo, H., Rischer, M. \& Poulsen, M. Natural products from microbes associated with insects. Beilstein J. Org. Chem. 12, 314-327 (2016).

14. Shi, Y.-M. \& Bode, H. B. Chemical language and warfare of bacterial natural products in bacteria-nematode-insect interactions. Nat. Prod. Rep. 35, 309-335 (2018).

15. Brachmann, A. O. et al. Pyrones as bacterial signaling molecules. Nat. Chem. Biol. 9, 573-578 (2013).

16. Brameyer, S., Kresovic, D., Bode, H. B. \& Heermann, R. Dialkylresorcinols as bacterial signaling molecules. Proc. Natl Acad. Sci. USA 112, 572-577 (2015).

17. Joyce, S. A. et al. Bacterial biosynthesis of a multipotent stilbene. Angew. Chem. Int. Ed. 47, 1942-1945 (2008).

18. Cai, X. et al. Entomopathogenic bacteria use multiple mechanisms for bioactive peptide library design. Nat. Chem. 9, 379-386 (2017).

19. Crawford, J. M., Portmann, C., Zhang, X., Roeffaers, M. B. J. \& Clardy, J. Small molecule perimeter defense in entomopathogenic bacteria. Proc. Natl Acad. Sci. USA 109, 10821-10826 (2012).

20. Theodore, C. M., King, J. B., You, J. \& Cichewicz, R. H. Production of cytotoxic glidobactins/luminmycins by Photorhabdus asymbiotica in liquid media and live crickets. J. Nat. Prod. 75, 2007-2011 (2012).

21. Lango-Scholey, L., Brachmann, A. O., Bode, H. B. \& Clarke, D. J. The expression of stlA in Photorhabdus luminescens is controlled by nutrient limitation. PLOS ONE 8, e82152 (2013).

22. Kontnik, R., Crawford, J. M. \& Clardy, J. Exploiting a global regulator for small molecule discovery in Photorhabdus luminescens. ACS Chem. Biol. 5, 659-665 (2010).

23. Wright, P. R. et al. CopraRNA and IntaRNA: predicting small RNA targets, networks and interaction domains. Nucleic Acids Res. 42, W119-W123 (2014).

24. Joyce, S. A. \& Clarke, D. J. A hexA homologue from Photorhabdus regulates pathogenicity, symbiosis and phenotypic variation. Mol. Microbiol. 47, 1445-1457 (2003)

25. Bouvier, M., Sharma, C. M., Mika, F., Nierhaus, K. H. \& Vogel, J. Small RNA binding to $5^{\prime}$ mRNA coding region inhibits translational initiation. Mol. Cell 32, 827-837 (2008).

26. Sharma, C. M., Darfeuille, F., Plantinga, T. H. \& Vogel, J. A small RNA regulates multiple $\mathrm{ABC}$ transporter mRNAs by targeting $\mathrm{C} / \mathrm{A}$-rich elements inside and upstream of ribosome-binding sites. Genes Dev. 21 2804-2817 (2007).

27. Yang, Q., Figueroa-Bossi, N. \& Bossi, L. Translation enhancing ACA motifs and their silencing by a bacterial small regulatory RNA. PLoS Genet. 10, e1004026 (2014)

28. Wenger, R. H. Mammalian oxygen sensing, signalling and gene regulation. J. Exp. Biol. 203, 1253-1263 (2000).

29. Chen, J. J. \& London, I. M. Regulation of protein synthesis by heme-regulated eIF-2 $\alpha$ kinase. Trends Biochem. Sci. 20, 105-108 (1995).

30. Qi, Z., Hamza, I. \& O'Brian, M. R. Heme is an effector molecule for iron-dependent degradation of the bacterial iron response regulator (Irr) protein. Proc. Natl Acad. Sci. USA 96, 13056-13061 (1999).

31. Rao, A. U., Carta, L. K., Lesuisse, E. \& Hamza, I. Lack of heme synthesis in a free-living eukaryote. Proc. Natl Acad. Sci. USA 102, 4270-4275 (2005).

32. Bolla, R. Developmental nutrition of nematodes: the biochemical role of sterols, heme compounds, and lysosomal enzymes. J. Nematol. 11, 250-259 (1979)

33. Bode, E. et al. Promoter activation in $\Delta h f q$ mutants as an efficient tool for specialized metabolite production enabling direct bioactivity testing. Angew. Chem. Int. Ed. 58, 18957-18963 (2019).

34. Zeng, Q., McNally, R. R. \& Sundin, G. W. Global small RNA chaperone Hfq and regulatory small RNAs are important virulence regulators in Erwinia amylovora. J. Bacteriol. 195, 1706-1717 (2013). 
35. Mukherjee, A., Cui, Y., Ma, W., Liu, Y. \& Chatterjee, A. K. hexA of Erwinia carotovora ssp. carotovora strain Ecc71 negatively regulates production of RpoS and rsmB RNA, a global regulator of extracellular proteins, plant virulence and the quorum-sensing signal, $\mathrm{N}$-(3-oxohexanoyl)-L-homoserine lactone. Environ. Microbiol. 2, 203-215 (2000).

36. Matilla, M. A., Leeper, F. J. \& Salmond, G. P. C. Biosynthesis of the antifungal haterumalide, oocydin A, in Serratia, and its regulation by quorum sensing, RpoS and Hfq. Environ. Microbiol. 17, 2993-3008 (2015).

37. Wilf, N. M. \& Salmond, G. P. C. The stationary phase sigma factor, RpoS, regulates the production of a carbapenem antibiotic, a bioactive prodigiosin and virulence in the enterobacterial pathogen Serratia sp. ATCC 39006. Microbiology 158, 648-658 (2012).

38. Wilf, N. M. et al. The RNA chaperone, Hfq, controls two luxR-type regulators and plays a key role in pathogenesis and production of antibiotics in Serratia sp. ATCC 39006. Environ. Microbiol. 13, 2649-2666 (2011).

39. Shanks, R. M. Q. et al. Suppressor analysis of eepR mutant defects reveals coordinate regulation of secondary metabolites and serralysin biosynthesis by EepR and HexS. Microbiology 163, 280-288 (2017).

40. White, G. F. A method for obtaining infective nematode larvae from cultures Science 66, 302-303 (1927).

41. Goodman, A. L. et al. Identifying genetic determinants needed to establish a human gut symbiont in its habitat. Cell Host Microbe 6, 279-289 (2009).

42. Brachmann, A. O. et al. A type II polyketide synthase is responsible for anthraquinone biosynthesis in Photorhabdus luminescens. Chembiochem 8, 1721-1728 (2007).

43. Bankevich, A. et al. SPAdes: a new genome assembly algorithm and its applications to single-cell sequencing. J. Comput. Biol. 19, 455-477 (2012).

44. Seemann, T. Prokka: rapid prokaryotic genome annotation. Bioinformatics 30 2068-2069 (2014).

45. Bolger, A. M., Lohse, M. \& Usadel, B. Trimmomatic: a flexible trimmer for Illumina sequence data. Bioinformatics 30, 2114-2120 (2014).

46. Langmead, B. \& Salzberg, S. L. Fast gapped-read alignment with Bowtie 2. Nat. Methods 9, 357-359 (2012).

47. Li, H. et al. The Sequence Alignment/Map format and SAMtools. Bioinformatics 25, 2078-2079 (2009).

48. Liao, Y., Smyth, G. K. \& Shi, W. featureCounts: an efficient general purpose program for assigning sequence reads to genomic features. Bioinformatics 30, 923-930 (2014).

49. Fröhlich, K. S., Haneke, K., Papenfort, K. \& Vogel, J. The target spectrum of SdsR small RNA in Salmonella. Nucleic Acids Res. 44 10406-10422 (2016)

50. Gibson, D. G. Synthesis of DNA fragments in yeast by one-step assembly of overlapping oligonucleotides. Nucleic Acids Res. 37, 6984-6990 (2009).

51. Dunn, A. K., Millikan, D. S., Adin, D. M., Bose, J. L. \& Stabb, E. V. New $r f p$ - and pES213-derived tools for analyzing symbiotic Vibrio fischeri reveal patterns of infection and lux expression in situ. Appl. Environ. Microbiol. 72 802-810 (2006).

52. Corcoran, C. P. et al. Superfolder GFP reporters validate diverse new mRNA targets of the classic porin regulator, MicF RNA. Mol. Microbiol. 84, 428-445 (2012).
53. Tobias, N. J., Linck, A. \& Bode, H. B. Natural product diversification mediated by alternative transcriptional starting. Angew. Chem. Int. Ed. 57, 5699-5702 (2018).

54. Hilker, R. et al. ReadXplorer 2-detailed read mapping analysis and visualization from one single source. Bioinformatics 32, 3702-3708 (2016).

55. Smith, C., Heyne, S., Richter, A. S., Will, S. \& Backofen, R. Freiburg RNA Tools: a web server integrating INTARNA, EXPARNA and LOCARNA. Nucleic Acids Res. 38, W373-W377 (2010).

56. Hakobyan, A., Liesack, W. \& Glatter, T. Crude-MS strategy for in-depth proteome analysis of the methane-oxidizing Methylocystis sp. strain SC2. J. Proteome Res. 17, 3086-3103 (2018).

57. Glatter, T. et al. Large-scale quantitative assessment of different in-solution protein digestion protocols reveals superior cleavage efficiency of tandem Lys-C/trypsin proteolysis over trypsin digestion. J. Proteome Res. 11, 5145-5156 (2012).

58. Tobias, N. J. et al. Cyclo(tetrahydroxybutyrate) production is sufficient to distinguish between Xenorhabdus and Photorhabdus isolates in Thailand. Environ. Microbiol. 121, 2921-2932 (2019).

\section{Acknowledgements}

This work was funded in part by the DFG (SFB 902, project no. B17) and the LOEWE Centre for Translational Biodiversity Genomics (LOEWE TBG), supported by the State of Hesse. K.P. acknowledges funding by the DFG (EXC 2051, grant no. 390713860), Vallee Foundation and European Research Council (grant no. StG-758212). We thank A. Goodman for providing pSAM-BT and for helpful discussions. We thank L. Pöschel and A. K. Heinrich for the plasmid construction.

\section{Author contributions}

N.N., N.J.T., M.H., X.C., A.L.L., T.G. and S.J.P. performed the experiments, except sequencing of the transposon-insertion mutants, which was performed by S.J.P. and T.P.S. N.N., N.J.T., M.H., K.P. and H.B.B. designed the study, discussed the results and commented on the manuscript. N.N., N.J.T. and M.H. analysed and interpreted the data. N.N., N.J.T. and M.H. wrote the manuscript. All of the authors read and approved the final manuscript.

\section{Competing interests}

The authors declare no competing interests.

\section{Additional information}

Extended data is available for this paper at https://doi.org/10.1038/s41564-020-00797-5. Supplementary information is available for this paper at https://doi.org/10.1038/ s41564-020-00797-5.

Correspondence and requests for materials should be addressed to H.B.B.

Reprints and permissions information is available at www.nature.com/reprints. Publisher's note Springer Nature remains neutral with regard to jurisdictional claims in published maps and institutional affiliations.

(c) The Author(s), under exclusive licence to Springer Nature Limited 2020 

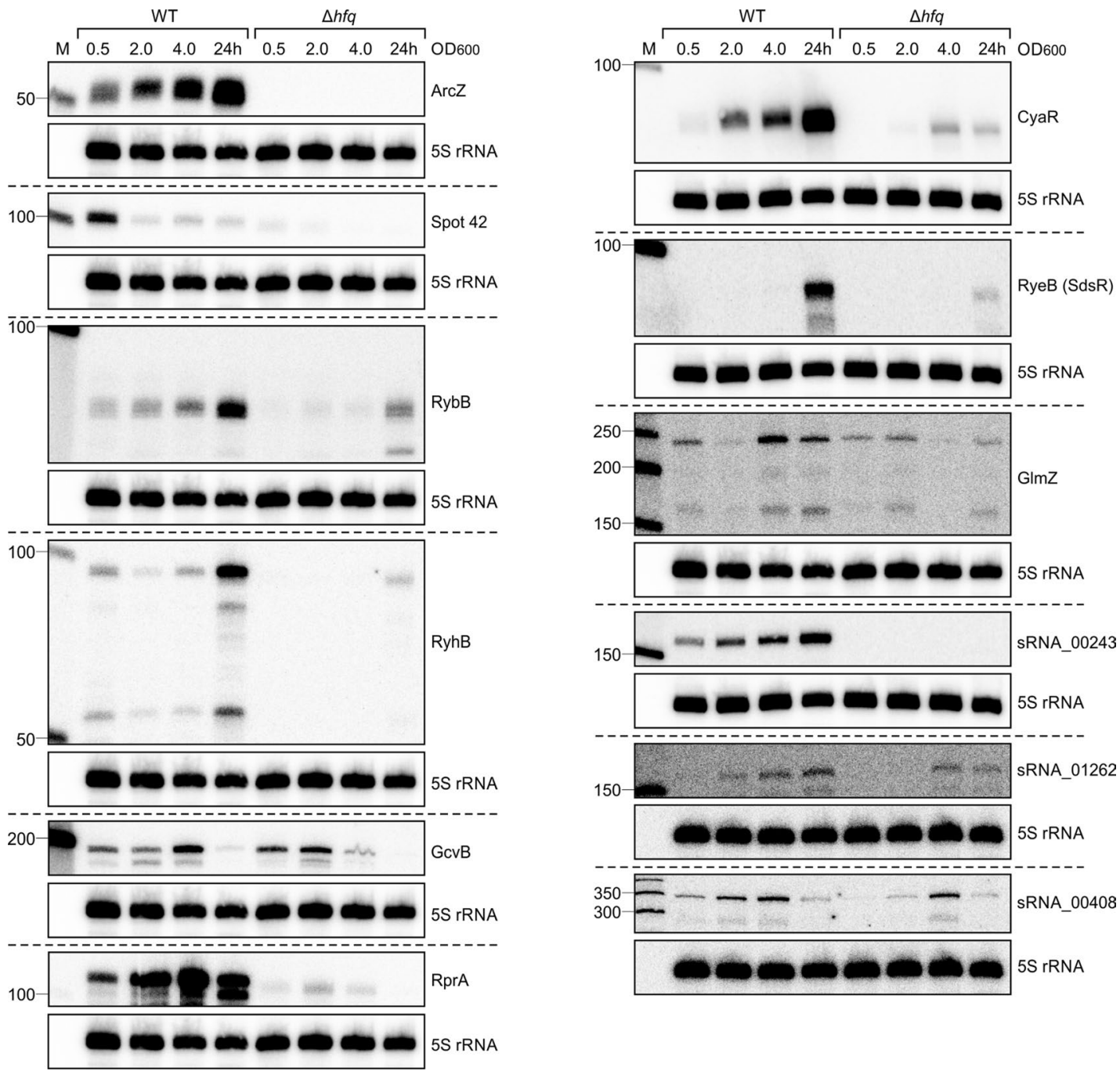

Extended Data Fig. 1 | Northern blot analysis of various sRNAs in P. laumondii. Expression of various sRNAs in P. laumondii at different time points. RNA samples of $P$. laumondii WT and $\Delta h f q$ strains were taken at three different $\mathrm{OD}_{600}$ values $(0.5,2$ and 4$)$ and after $24 \mathrm{~h}$ of growth. The RNA was loaded on northern blots and probed for the indicated sRNAs. Probing for $5 \mathrm{~S}$ rRNA served as loading control. 

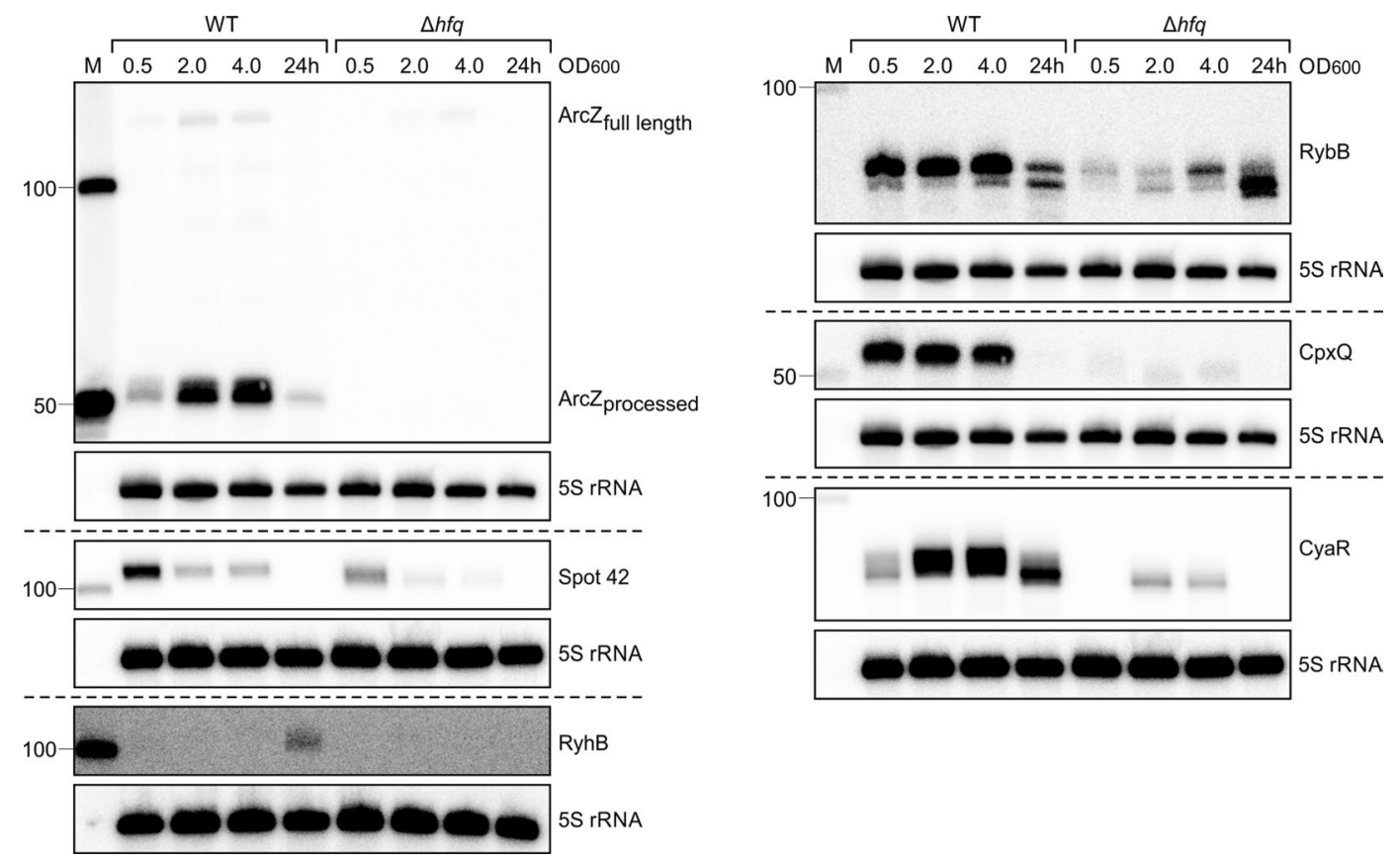

Extended Data Fig. 2 | Northern blot analysis of various sRNAs in X. szentirmaii. Expression of various sRNAs in X. szentirmaii at different time points. RNA samples of $X$. szentirmaii WT and $\Delta h f q$ strains were taken at three different $\mathrm{OD}_{600}$ values $(0.5,2$ and 4$)$ and after $24 \mathrm{~h}$ of growth. The RNA was loaded on northern blots and probed for the indicated sRNAs. Probing for $5 \mathrm{~S}$ rRNA served as loading control. 
a

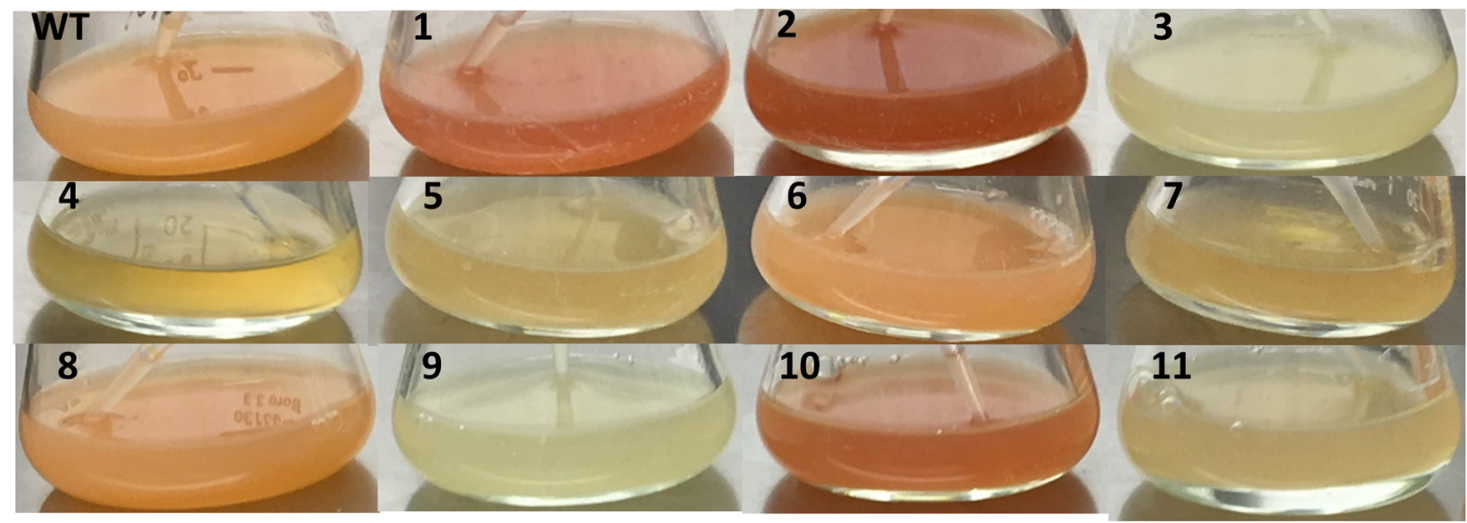

b

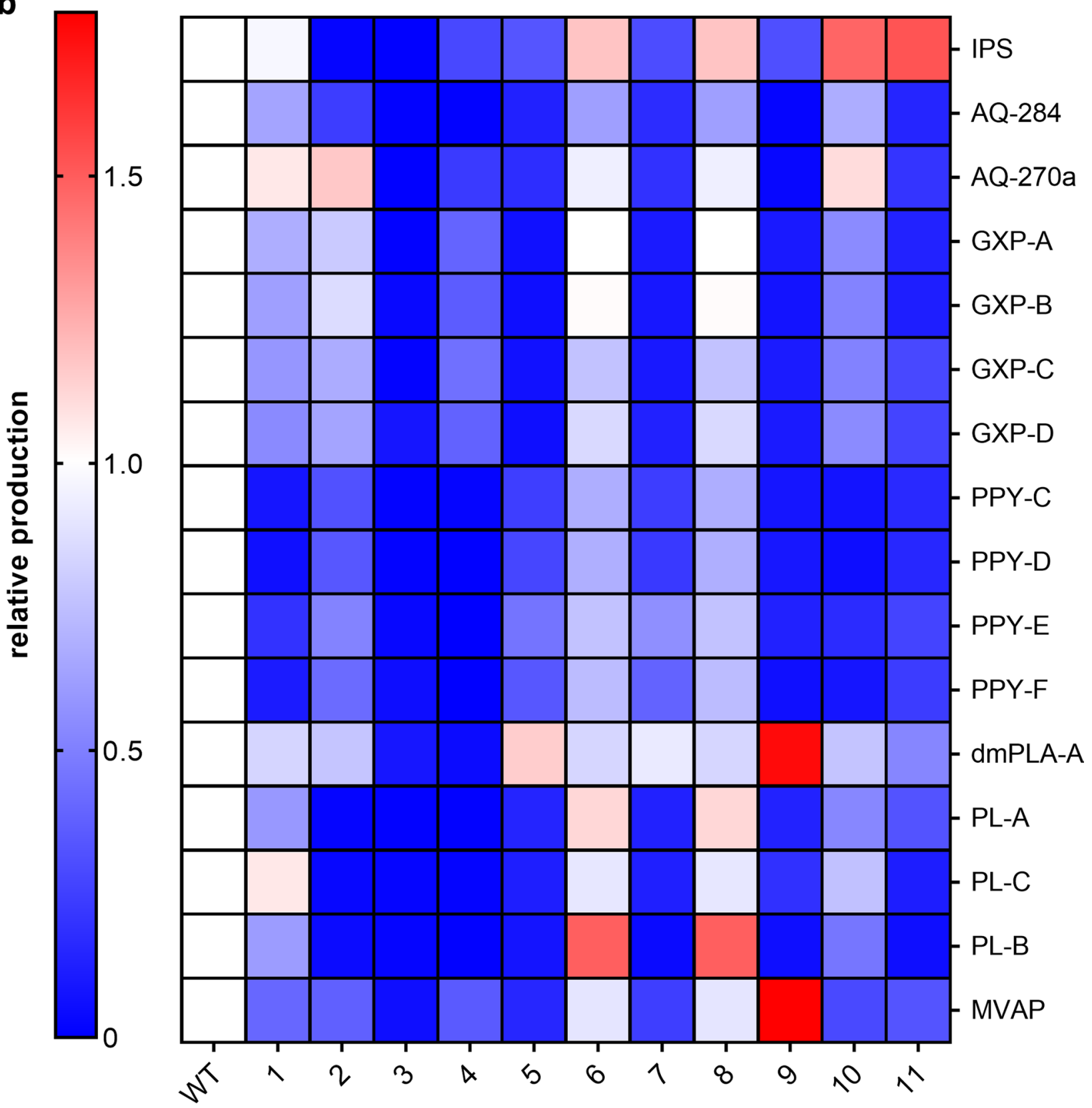

Extended Data Fig. 3 | See next page for caption. 
Extended Data Fig. 3 | Phenotype and specialized metabolite profile of transposon-insertion mutants of $P$. laumondii. Phenotype of transposon-insertion mutants of $P$. laumondii. a. Differences in pigmentation of transposon-insertion mutant liquid cultures compared to WT. Depicted are eleven

transposon-insertion mutants and a WT culture after $3 \mathrm{~d}$ of cultivation at $30^{\circ} \mathrm{C}$ with shaking. $\mathbf{b}$. SM profiles of the transposon-insertion mutants. Relative SM production was quantified from duplicates using TargetAnalysis (Bruker) and compared to the WT of $P$. laumondii after $72 \mathrm{~h}$ cultivation at $30^{\circ} \mathrm{C}$ with shaking. Mutant 3 was analysed further and the transposon insertion was identified in the $\operatorname{arc} Z$ gene. 


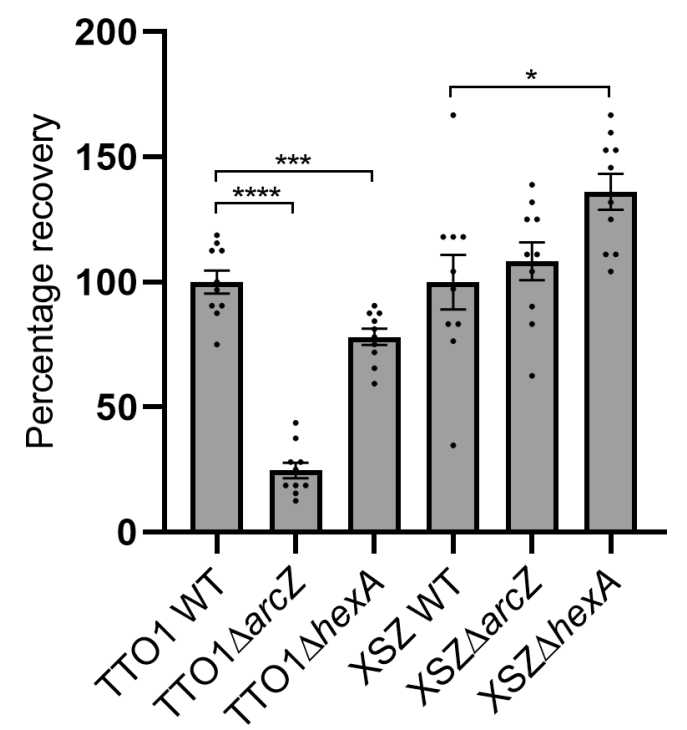

Extended Data Fig. 4 | Nematode bioassay. Infective juvenile development to hermaphrodites with strains of $P$. laumondii and $X$. szentirmaii. Data are presented as the mean \pm s.e.m. Dots represent biologically independent replicates $(n=10)$. Asterisks indicate statistical significance $\left({ }^{\star} P<0.05\right.$, $\left.{ }^{\star \star} P<0.005,{ }^{\star \star \star} P<0.0005,{ }^{\star \star \star \star} P<0.00005\right)$ of relative recovery compared to WT recovery levels. Statistical significances were calculated using a two-sided unpaired t-test. Exact $p$ values (left to right, respectively) for P. laumondii TTO1 correspond to $p=<0.0001,0.0006$ and for $X$. szentirmaii to $P=0.56,0.0094$. 


\section{Reporting Summary}

Nature Research wishes to improve the reproducibility of the work that we publish. This form provides structure for consistency and transparency in reporting. For further information on Nature Research policies, see Authors \& Referees and the Editorial Policy Checklist.

\section{Statistics}

For all statistical analyses, confirm that the following items are present in the figure legend, table legend, main text, or Methods section.

$\mathrm{n} / \mathrm{a}$ | Confirmed

$\square$ \The exact sample size $(n)$ for each experimental group/condition, given as a discrete number and unit of measurement

$\square$ \A statement on whether measurements were taken from distinct samples or whether the same sample was measured repeatedly

The statistical test(s) used AND whether they are one- or two-sided

$\square$ Only common tests should be described solely by name; describe more complex techniques in the Methods section.

Х $\square$ A description of all covariates tested

Х $\square$ A description of any assumptions or corrections, such as tests of normality and adjustment for multiple comparisons

A full description of the statistical parameters including central tendency (e.g. means) or other basic estimates (e.g. regression coefficient)

AND variation (e.g. standard deviation) or associated estimates of uncertainty (e.g. confidence intervals)

$\square$ For null hypothesis testing, the test statistic (e.g. $F, t, r$ ) with confidence intervals, effect sizes, degrees of freedom and $P$ value noted

$\square$ Give P values as exact values whenever suitable.

Х $\square$ For Bayesian analysis, information on the choice of priors and Markov chain Monte Carlo settings

Х $\square$ For hierarchical and complex designs, identification of the appropriate level for tests and full reporting of outcomes

Х $\square$ Estimates of effect sizes (e.g. Cohen's $d$, Pearson's $r$ ), indicating how they were calculated

Our web collection on statistics for biologists contains articles on many of the points above.

\section{Software and code}

Policy information about availability of computer code

Data collection During HPLC/MS anaylsis, Chromeleon Chromatography Data System (CDS) was used to collect HPLC data and HyStar (v 3.2) for MS data.

Data analysis Genomes were assembled with SPAdes (v3.10.1) and annotated with Prokka (v1.12). Completed genome sequences were analysed and viewed in Geneious v9.1 and v6. Raw sequencing data was trimmed using Trimmomatic (v0.39), mapped using bowtie (v2.3.4.3) converted to bam using samtools (1.8) and counted using featureCounts (a part of the subread package (v1.6.5)). Statistical analysis was performed in $\mathrm{R}$ ( $\mathrm{v}$ 3.6.1) on the degust platform (http://degust.erc.monash.edu/), where exact code is available to view. Transcriptional start sites were detected using readXplorer (V2.2.3). CopraRNA (v2.1.2) was used to predict ArcZ binding sites. Chemical compound analysis was performed using The compounds peak areas were quantified using TargetAnalysis (v1.3). Label-free quantification was performed using the Progenesis QI software (Nonlinear Dynamics, v2.0). Progenesis output was processed with SafeQuant (v2.3.1).

For manuscripts utilizing custom algorithms or software that are central to the research but not yet described in published literature, software must be made available to editors/reviewers. We strongly encourage code deposition in a community repository (e.g. GitHub). See the Nature Research guidelines for submitting code \& software for further information.

\section{Data}

Policy information about availability of data

All manuscripts must include a data availability statement. This statement should provide the following information, where applicable:

- Accession codes, unique identifiers, or web links for publicly available datasets

- A list of figures that have associated raw data

- A description of any restrictions on data availability

All .mzXML files from HPLC-MS runs are available at MassIVE (https://massive.ucsd.edu) under the ID MSV000084163. Raw sequence data is available at the European nucleotide archive (https://www.ebi.ac.uk/ena/) under project accession numbers PRJEB33827 and PRJEB24159. The proteomic data can be accessed at PRIDE (https://www.ebi.ac.uk/pride/) with the project accession number PXD019095. Source Data are provided with this paper. 


\section{Field-specific reporting}

Please select the one below that is the best fit for your research. If you are not sure, read the appropriate sections before making your selection. \Life sciences

$\square$ Behavioural \& social sciences Ecological, evolutionary \& environmental sciences

For a reference copy of the document with all sections, see nature.com/documents/nr-reporting-summary-flat.pdf

\section{Life sciences study design}

All studies must disclose on these points even when the disclosure is negative.

Sample size For sequencing experiments, duplicates were used. Based on our previous experience this has been sufficient to determine significant differences using strict false-discovery cutoffs (https://sfamjournals.onlinelibrary.wiley.com/doi/full/10.1111/1462-2920.13502; https:// www.nature.com/articles/s41589-019-0246-1\#Sec10). Chemical compound analyses were performed in triplicate to confirm observations. Based on our experience, the relative production of natural products throughout replicates does not fluctuate very much, as also indicated by the standard deviations shown in this paper and previous work (https://pubmed.ncbi.nlm.nih.gov/28261170/). Nematode bioassays were performed in ten replicates. Sample sizes for nematode bioassays were chosen according to previously published work (https:// sfamjournals.onlinelibrary.wiley.com/doi/full/10.1111/1462-2920.13502).

Data exclusions No data were excluded from analysis.

Replication All sequencing experiments were performed in duplicate followed by normalization and statistical analyses. We confirmed several outcomes defined by predictive tools by using molecular methods (ie. specific DNA modifications). Nematode bioassays and compound analyses were performed independently, in triplicate to confirm observations.

Randomization All experiments were designed to elucidate a specific functional mechanism. Randomization was not relevant to this study because there was no group allocation involved.

Blinding

Blinding was not relevant to this study. The majority of sequencing experiments were RNA based and the results therefore are definitive. No modification of any data was performed and all results are reported as direct outputs of individual analyses.

\section{Reporting for specific materials, systems and methods}

We require information from authors about some types of materials, experimental systems and methods used in many studies. Here, indicate whether each material, system or method listed is relevant to your study. If you are not sure if a list item applies to your research, read the appropriate section before selecting a response.

Materials \& experimental systems

n/a Involved in the study

$\square$ Antibodies

$\bigotimes \square$ Eukaryotic cell lines

$\bigotimes \square$ Palaeontology

$\bigotimes \square$ Animals and other organisms

$\bigotimes \square$ Human research participants

$\bigotimes \square$ Clinical data

\section{Antibodies}

Antibodies used

Monoclonal ANTI-FLAG M2 antibody, Sigma, \#F1804, Lot\# SLBT7654, produced in mouse

Validation

commercially available antibody; purified IgG1 monoclonal antibody that binds to fusion proteins containing a FLAG peptide sequence

\begin{tabular}{l|l} 
Methods \\
\hline n/a & Involved in the study \\
\hline & $\square$ ChIP-seq \\
$\square$ & $\square$ Flow cytometry \\
$\square$ & $\square$ MRI-based neuroimaging
\end{tabular}

